Aufsatz

Joachim Krause*

\title{
Die Nordatlantische Allianz und Abschreckung durch Kernwaffen
}

https://doi.org/10.1515/sirius-2021-4005

Kurzfassung: Die Nordatlantische Allianz wird bei der Abfassung eines neuen strategischen Konzepts nicht die Frage ausklammern können, welche Rolle Kernwaffen zur Abschreckung einer möglichen Invasion Russlands im Ostseeraum (oder auch im Schwarzmeerraum) zukommen wird. Diese Diskussion muss sich an der aktuellen Bedrohung orientieren und muss offen und ehrlich darlegen, weshalb eine Kriegsverhinderung weiterhin die europäische Teilhabe an der nuklearen Abschreckungsmacht der NATO erfordert. Dabei müssen die Natur der durch Russland verursachten Bedrohung für NATO-Mitglieder aufgezeigt und die Rolle von eurostrategischen Kernwaffen Russlands deutlich benannt werden. Nur so wird es möglich sein, die Rolle von amerikanischen Kernwaffen in Europa sowie die Bedeutung der Verfahren und Instrumente einer nuklearen Teilhabe klar einzuschätzen.

Schlüsselbegriffe: NATO, Abschreckung, Kernwaffen

\begin{abstract}
In drafting a new Strategic Concept, the North-Atlantic Alliance will have to address the issue of nuclear deterrence against possible Russian attempts to invade and occupy member states of NATO in the Baltic or in the Black Sea-area. This debate must start from assessing the nature of the threat (both conventional and nuclear) and it has to be clear about the nuclear component as part of NATO's deterrent strategy. In this sense, it is important that the role of nuclear weapons in Russia's strategy for regional wars is being examined. Only by looking at the subject from the threat-side, a reasonable narrative for the continuation of nuclear sharing arrangements can be formulated.
\end{abstract}

Keywords: NATO, deterrence, nuclear weapons

Kontakt: Prof. Dr. Joachim Krause, Direktor, Institut für Sicherheitspolitik an der Universität Kiel, Geschäftsführender Herausgeber von Sirius, E-Mail: jkrause@politik.uni-kiel.de

\section{Einleitung}

Seit ihrer Gründung beruht die Nordatlantische Allianz auf der Idee der Abschreckung gegen militärische Aggressionen, wobei (amerikanischen) Kernwaffen stets eine zentrale Rolle zukam und weiterhin zukommt. Diese Rolle hat sich zwar im Lauf der Zeit geändert, aber die zentrale Funktion von Kernwaffen für den Zusammenhalt der Allianz wurde und wird immer wieder herausgestellt, so im Strategischen Konzept der NATO aus dem Jahr 2010, bei der Überprüfung des Abschreckungs- und Verteidigungskonzepts der Allianz 2012 und zuletzt beim NATO-Gipfel im Juli 2021. ${ }^{1}$ In der Vergangenheit ging es hauptsächlich um Kernwaffen der USA, die Aggressionen der Sowjetunion gegen die europäischen Verbündeten verhindern sollten. Ziel war die Kriegsvermeidung oder (sofern ein Kriegsausbruch sich nicht verhindern ließ) die rasche Kriegsbeendigung zu annehmbaren Bedingungen. Die westliche Staatengemeinschaft wollte damit die Implementierung des Gewaltverbots der UN-Charter garantieren helfen, aber auch Vorsorge treffen für den Fall, dass sich Großmächte wie die Sowjetunion oder heute Russland nicht an das Gewaltverbot halten. ${ }^{2}$ Abschreckung zielt immer darauf ab, das Risikokalkül der anderen Seite so zu beeinflussen, dass es zu keinem Krieg kommt, sie ist also eine mentale Angelegenheit (cosa mentale). Sie bedarf jedoch, um wirksam zu sein, der Unterfütterung durch militärische Potentiale, die Kriegführung und Bestrafung ermöglichen. ${ }^{3}$

Die physikalischen Eigenschaften von Kernwaffen galten und gelten als dermaßen beispiellos, dass man ihnen für die Abschreckung eine zentrale Rolle zumaß. Allerdings waren es gerade die Folgen des Einsatzes von

1 Kamp 2018, Roberts 2021a, 2 und 3, siehe auch de Wijk 2018, 6-10. Im Kommuniqué des NATO-Gipfeltreffens vom Juni 2021 wurde in Paragraph 40 erneut betont, dass die NATO eine nukleare Allianz bleibt, solange es Kernwaffen in der Welt gibt, Text des Kommuniqués auf der Webseite der NATO: https://www.nato.int/cps/en/natohq/ news_185000.htm?selectedLocale=en

2 Bergeron 2018, 22; Thomson 2018, 32.

3 Delpech 2012, 23. 
Kernwaffen, die Kritik an der nuklearen Abschreckung entzündeten. Die Bilder der Zerstörung von Hiroshima und Nagasaki waren und sind für viele Menschen Anstoß, Kernwaffen gänzlich abzulehnen. ${ }^{4}$ Und die Entwicklung der nuklearstrategischen Konkurrenz zwischen den USA und der Sowjetunion in den 60er, 70er und 80er Jahren des 20. Jahrhunderts trug dazu bei, dass die Risiken eines nuklearen Rüstungswettlaufes ins Visier gerieten und Überlegungen zur Rüstungskontrolle angestellt wurden. ${ }^{5}$ Außerdem gab es von Anbeginn an einen grundsätzlichen Dissens darüber, was „abschreckend“ wirkt: allein der Besitz von Kernwaffen oder die Fähigkeit, sie in überlegter Weise einzusetzen und so einen Krieg mit Kernwaffen erfolgreich führen zu können. ${ }^{6}$

Nachdem die politische - und damit auch die militärische - Bedeutung von Kernwaffen in den vergangenen drei Jahrzehnten abgenommen hatte, vollzieht sich derzeit ein Wandel: Aufgrund der zunehmend konfrontativen internationalen Beziehungen werden Kernwaffen nach Ansicht vieler Beobachter wieder wichtig. ${ }^{7}$ Gleichzeitig verbreitert sich das Spektrum der Domänen, in denen Abschreckung praktiziert werden kann und muss. ${ }^{8}$ Bei der anstehenden Ausarbeitung eines neuen strategischen Konzepts der NATO wird die Diskussion über das Gewicht von Kernwaffen bei der Wiederherstellung eines Abschreckungs- und Verteidigungsdispositivs gegen regionale russische Aggressionen dennoch eine zentrale Rolle spielen müssen. ${ }^{9}$ Hinzu kommen Chinas Aufrüstung und dessen geostrategische Zusammenarbeit mit Russland. Die Schlussfolgerungen müssen die erwähnten moralischen Bedenken und die Risikokalküle bezüglich eventueller Rüstungswettlaufrisiken berücksichtigen, aber sie müssen sich auch daran messen lassen, in welchem Maß sie Antworten auf real existierende Bedrohungen liefern oder nicht. Diese Diskussion wird besonders in Deutschland politisch schwer zu führen sein und erfordert von den politisch Verantwortlichen sehr viel Mut. Viele Stimmen aus Politik und Wissenschaft bezweifeln die Notwendigkeit von Kernwaffen. Insbesondere gilt die nukleare Teilhabe der Bundeswehr als überflüssig, ebenso die Lagerung amerikanischer Kernwaffen auf dem Boden Deutschlands und anderer europäischer NATO-Staaten (u. a. Niederlande, Belgien, Italien). Die angeführten Argumente reflektieren allerdings häufig eine sehr oberflächliche Auseinandersetzung mit der The- matik und greifen gern auf US-kritische Einstellungsmuster und friedenspolitische Vereinfachungen zurück. Da sie in der politischen Debatte großen Widerhall finden, ist es umso notwendiger, die aufgeworfenen Fragen gründlich und abgewogen zu behandeln.

Im Folgenden sollen die wesentlichen Voraussetzungen und Kontroversen aufgezeigt und mögliche Lösungswege diskutiert werden. Den Anfang macht ein Rückblick auf die Rolle von Kernwaffen in der Zeit des Ost-WestKonflikts. Nur so ist es möglich, die heutige Lage von der Vergangenheit abzugrenzen, Gemeinsamkeiten und Unterschiede herauszuarbeiten. Es folgt eine Analyse der militärischen Bedrohungen, denen die europäischen Staaten der nordatlantischen Allianz derzeit durch Russland ausgesetzt und für deren Abschreckung Kernwaffen zu erwägen sind. Diese Grundlage erlaubt es, die Angemessenheit von strategischen Überlegungen oder Konzepten zu bewerten. ${ }^{10}$ Ausgehend von dieser Analyse wird nachgedacht über ein optimales nukleares Abschreckungsdispositiv, das der Bedrohung gerecht wird und eine möglichst breite politische Akzeptanz findet. „Optimal“ bedeutet, dass es nie vollständige Sicherheit über das Funktionieren von „Abschreckung “ geben kann, insbesondere nicht über erweiterte nukleare Abschreckung (extended deterrence). Erweiterte Abschreckung meint, dass die USA zur Verteidigung ihrer europäischen Verbündeten im Fall eines russischen Angriffs glaubhaft bereit sind, den Krieg mit einem Kernwaffeneinsatz zu beenden - und zwar auch dann, wenn die USA selber Gegenstand eines russischen Vergeltungsschlags werden könnten. Daraus resultieren unterschiedliche und widersprüchliche Anforderungen an die US-Streitkräfte. ${ }^{11}$ Das Dilemma ist seit über 60 Jahren bekannt und wird sich nie vollständig auflösen lassen. ${ }^{12}$ Solange es keine tragfähige Alternative gibt, wird man damit leben müssen. Zumindest ist der Kalte Krieg nicht in einem Krieg (ob nuklear oder konventionell) geendet. Ob das der nuklearen Abschreckung geschuldet war oder nicht, lässt sich im Nachhinein nicht ergründen, doch man kann immerhin festhalten, dass das Wissen um die Risiken einer nuklearen Eskalation wesentlichen Anteil an der Vorsicht beider Seiten hatte und diesen strategischen Konflikt nicht in einen Weltkrieg münden ließ. ${ }^{13}$

10 Vgl. Krause 2015.

11 Vgl. Cordesman 1982.

12 Kissinger 1957, Kap. VI; Brodie 1958, 27; Schelling 1966, 35. 13 Delpech 2012, Kap. I.

4 Mandelbaum 1984.

5 Vgl. die Beiträge in Brennan 1961 sowie in Bull 1961.

6 De Wijk 2018, $4 \mathrm{f}$.

7 Delpech 2012, Nerlich 2020.

8 Bergeron 2018, de Wijk 2018, Thomson 2018, Mallory 2019.

9 Roberts 2015, 176-196. 


\section{Die Rolle von Kernwaffen zu Zeiten des Ost-West-Konflikts}

In den Jahren der militärischen Konfrontation zwischen der Sowjetunion und ihren verbündeten kommunistisch regierten Staaten einerseits und dem atlantischen Bündnis andererseits (1950-1989) besaßen Kernwaffen anfangs im amerikanischen Dispositiv - und somit auch dem der NATO - eine zentrale Stellung. Von Mitte 1950 dem Beginn des Koreakriegs - an galt die Gefahr einer bewaffneten Großinvasion Sowjetrusslands in Westeuropa angesichts der zahlenmäßigen Überlegenheit der Roten Armee als primäres Risiko europäischer Sicherheits- und Verteidigungspolitik. ${ }^{14}$ Kernwaffen waren ab 1954 bevorzugtes Mittel der Abschreckungspolitik, wobei den in und um Europa stationierten US-amerikanischen Bomberflugzeugen eine zentrale Rolle als Träger von Nuklearwaffen zukam. ${ }^{15}$ Im Rahmen der seit 1954 gültigen NATO-Strategie der „massiven Vergeltung“ (massive retaliation) kamen im Laufe der 50er Jahre die ersten taktischen Kernwaffen hinzu, die von ballistischen Raketen, Kampfflugzeugen oder per Artillerie verschossen werden konnten oder gar als Minen konstruiert waren. ${ }^{16}$ Sie sollten der sowjetischen Führung signalisieren, dass bereits ein begrenzter militärischer Übergriff auf eine nukleare Vergeltung treffen würde. Dieses recht simple Konstrukt einer Abschreckungsstrategie war so lange glaubwürdig, wie die USA die Sowjetunion mit Kernwaffen angreifen konnten, ohne selbst sowjetischen Vergeltungsschlägen ausgesetzt zu sein. Es war auch nur so lange glaubwürdig, wie die USA bei taktischen Kernwaffen das Monopol innehatten. Beide Voraussetzungen waren schon in den späten 50er und frühen 60er Jahren nicht mehr gegeben. Ab Ende der 50er Jahre verfügte auch das sowjetische Militär über nukleare Gefechtsfeldwaffen ${ }^{17}$ (und integrierte diese in ihre offensiv angelegte Militärdoktrin) und war nach und nach in der Lage, das Territorium der USA hauptsächlich mit landgestützten interkontinentalen Raketen zu bedrohen.

Diese rüstungstechnischen Entwicklungen erforderten eine Reform der NATO-Strategie, die - nicht zuletzt wegen des Widerstands der damaligen Bundesregierung -

14 Vgl. Cornides 1957, $224 \mathrm{ff}$.

15 Richardson 1967, $41 \mathrm{ff}$.

16 Vgl. für einen Überblick über die Potenziale der NATO und des Warschauer Paktes an taktischen Kernwaffen zu Beginn der 80er Jahre siehe Bracken 1983, 129-178 sowie Arkin/Fieldhouse 1985, 101-116.

17 Meyer 1987a und Meyer 1987b, 6-18.

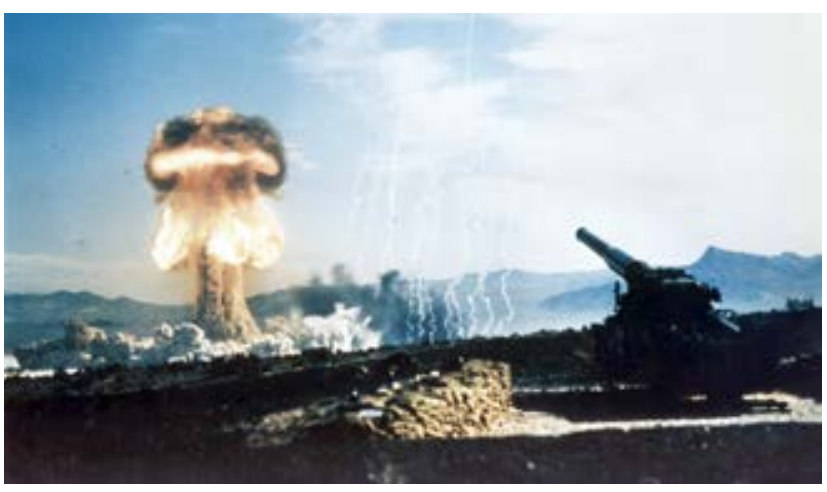

Test einer durch Artillerie verschossenen Nuklearwaffe in der Wüste von Nevada 1953

erst 1967 beschlossen und umgesetzt werden konnte. ${ }^{18}$ Laut der neuen NATO-Strategie der "flexiblen Antwort“ (flexible response) sollten taktische Kernwaffen nicht als erstes Mittel eingesetzt werden, sondern der Abschreckung des Gebrauchs nuklearer Gefechtsfeldwaffen seitens der Sowjetunion und des Warschauer Pakts dienen. ${ }^{19}$ Die zentrale Rolle räumte man vielmehr der mobilen Vorneverteidigung durch Verzögerungs-, Verteidigungs- und Gegenangriffsoperationen mittels konventioneller Streitkräfte ein und fasste dafür auch weitgehende Anstrengungen zur Verstärkung und Verbesserung der konventionellen Streitkräfte ins Auge.

Ein Gleichgewicht bei konventionellen Streitkräften in Mitteleuropa vermochten diese Bestrebungen nie herzustellen, doch war das Übergewicht der konventionellen Streitkräfte des Warschauer Paktes in den 70er und 80er Jahren nie so groß, dass ein Überfall auf Westdeutschland und Westeuropa ein leichtes Unterfangen gewesen wäre. ${ }^{20}$ Die in Westdeutschland befindlichen NATO-Truppen - darunter 12 Bundeswehrdivisionen - befanden sich in einem hohen Bereitschaftsstand und wären fähig gewesen, einer rein konventionellen Invasion des Warschauer Pakts für eine begrenzte Zeit standzuhalten, zumindest bis zum Ein-

$18 \mathrm{Zu}$ den Hintergründen vgl. Kissinger 1965, 153-219, Richardson 1967, 62-85, Mahncke 1972, Kelleher 1975, Haftendorn 1983, 156-206, Stromseth 1988, Haftendorn 1994.

19 Diese und die folgenden Überlegungen beruhen auf Stratmann 1981 und Rühl 1987 sowie Petersen/Hines 1983, Vigor 1983, Daalder 1991, siehe auch de Wijk 2018, 4-6.

20 Vgl. Stratmann 1981, 39-54; seiner Einschätzung nach war das Kräfteverhältnis nie so, dass der Warschauer Pakt sich sicher sein konnte, schnell einen Sieg davonzutragen. Die kriegsverhindernde Abschreckungswirkung der NATO sei stabil gewesen (227). Die beste Analyse zu den Kräfteverhältnissen zwischen NATO und Warschauer Pakt bei konventionellen Streitkräften finden sich bei Karber 1982, zu den nuklearen Kräfteverhältnissen bei Martin 1982. 
treffen umfangreicher Verstärkungen über den Atlantik. Möglicherweise hätte sich danach das Kriegsgeschehen zugunsten des Warschauer Pakts gewandelt, nämlich falls die NATO der zweiten strategischen Welle des Warschauer Pakts nichts Gleichwertiges mehr hätte entgegensetzen können.

Bereits ein konventionell geführter Krieg auf deutschem Boden hätte wenig übriggelassen von dem, was verteidigt werden sollte. Deshalb fand das Konzept der „vorbedachten Eskalation“ (premeditated escalation) Eingang in die einschlägigen NATO-Planungen der 70er und 80er Jahre. Es sah vor, im Falle einer absehbaren Niederlage der NATO in einem rein konventionell geführten Krieg der Sowjetunion durch Einsatz einzelner amerikanischer Atomwaffen (initial use) die Gefahr einer nuklearen Eskalation vor Augen zu führen. Sollte dieser erste Einsatz (vermutlich gegen eher symbolische Ziele außerhalb der Sowjetunion) zu keinem Erfolg führen, waren weitere einzelne nukleare Schläge geplant - auch gegen sowjetisches Territorium, aber nie bis zur Gefährdung sowjetischer strategischer Angriffskräfte (follow-onuse). Die Meinungen, wie die konkrete Einsatzplanung für den initial use und den follow-on use hätten aussehen sollen, waren unterschiedlich. Doch 1969 vereinbarte man vorläufige politische Richtlinien für den defensiven NATO-Einsatz taktischer Kernwaffen, die 1986 von allgemeinen politischen Richtlinien abgelöst wurden. ${ }^{21}$ Die Beratungen über diese Richtlinien machten die Interessenunterschiede zwischen der Bundesrepublik Deutschland (als Land, das am stärksten von Kampfhandlungen und dem Einsatz von nuklearen Gefechtsfeldwaffen betroffen wäre) und den USA deutlich. Die Bundesregierung legte darauf Wert, dass konventionelle Kampfhandlungen auf deutschem Boden nicht zu lange dauern und die USA sich zu möglichst frühen nuklearen Einsätzen verpflichten sollte, die sowjetisches Territorium nicht aussparten. Washington hingegen wollte sich möglichst viele Optionen offenhalten. Doch am wichtigsten war: Man konnte sich auf gemeinsame Richtlinien einigen, die diese Differenzen überbrückten. Besonders heikel war das Thema „Gebrauch von taktischen Gefechtsfeldwaffen“ für den Fall, dass der Warschauer Pakt diese zuerst eingesetzt hätte. Hier klafften die Ansichten der deutschen und amerikanischen Vertreter noch weiter auseinander, da ein Einsatz taktischer Gefechtsfeldwaffen auf deutschem Boden hätte

21 Vgl. Daalder 1991, 90-93, Hoppe 1993, Bluth 1995, 261-263, Halverson 1995, 70-76, Heuser 1997, 57-60, Heuser 2006, 211. Siehe unter der neueren Literatur vor allem Lutsch 2020, Kapitel V, der die damaligen Debatten unter Einbeziehung heute einsehbarer Regierungsdokumente gründlich neu aufgearbeitet hat. stattfinden müssen mit entsprechend katastrophalen Kollateralschäden.22

Weitere Interessensunterschiede bestanden bei diesem Thema mit Frankreich: Seine landgestützten Nuklearwaffen auf dem Plateau d'Albion hätte man gegen die Streitkräfte des Warschauer Pakts einsetzen müssen, wenn diesen als Zwischenziel der Durchbruch zum Rhein gelungen wäre. Daher hätte sich die Bundesrepublik auch mit Blick auf Frankreich keine Niederlage in einer konventionellen Abwehrschlacht erlauben können.

Auf Seiten der Sowjetunion und des Warschauer Pakts kalkulierte man anders. Zum einen wollte die sowjetische Führung das Territorium der USA weitgehend mit interkontinentalen Raketenwaffen bedrohen können. Die strategische Konkurrenz mit den USA war Kern der sowjetischen militärischen Anstrengungen. Gleichzeitig ließ sich damit in den Augen der europäischen Alliierten der USA, insbesondere der Bundesrepublik Deutschland, die Glaubwürdigkeit der amerikanischen erweiterten Abschreckung erschüttern. Zweitens sahen die Militärdoktrin der Sowjetunion und die operativen Einsatzplanungen des Warschauer Pakts für einen Kriegsschauplatz in Europa vor, die eigenen Streitkräfte sofort in die landgreifende Offensive gehen zu lassen und dabei auch nukleare Gefechtsfeldwaffen einzusetzen, die man als besonders geeignet für das Erzwingen großer Durchbrüche ansah. ${ }^{23}$

Neben der neuen NATO-Strategie der flexiblen Verteidigung wurde 1967 das Programm des Harmel-Berichts beschlossen, das unter anderem vorsah, unter der Bedingung eines gesicherten militärischen Gleichgewichts der Sowjetunion und dem Warschauer Pakt Gespräche über alle strittigen politischen Fragen anzubieten. ${ }^{24}$ Dahinter stand auch das Bestreben der Johnson-Administration in Washington, mit der sowjetischen Führung in einen Dialog über nuklearstrategische Stabilität einzutreten. ${ }^{25}$ In den 60er Jahren hatten sowohl die USA als auch die Sowjetunion nuklear bewaffnete Interkontinentalraketen aufgestellt und begannen zudem mit der Entwicklung von Raketensystemen, die anfliegende Interkontinentalraketen der jeweils anderen Seite im Weltraum zerstören konnten (Anti-Ballistic-Missiles - ABM). Vor allem aus der akademischen Welt war wiederholt darauf hingewiesen worden, dass ein unkontrollierter Verlauf der nuklearstrategischen Rüstung gefährliche Situationen provozie-

22 Vgl. Bracken 1983, 164 ff., s. a. Charles 1987 sowie Kelleher 1988. 23 Vgl. Nielsen 1998, Mastny 2006, Lunak 2001, Lunak 2006, Lautsch 2013.

24 Zum Harmel-Bericht vgl. Haftendorn 1992, Bozo 1998 sowie Rynning 2017.

$25 \mathrm{Vgl}$. Windsor 1971, $13 \mathrm{ff}$. 
ren könnte. ${ }^{26}$ Es bestand die Gefahr eines politisch nicht autorisierten Einsatzes von Kernwaffen (wie bei der KubaKrise 1962) oder dass ein Zwischenfall, Missverständnisse oder Informationsmängel einen Kernwaffenkrieg auslösen könnten. Vor allem ging es um Krisenstabilität: Richteten beide Staaten in der Hauptsache landgestützte Interkontinentalraketen aufeinander, stand zu befürchten, die eine oder die andere Seite könnte im Verlauf einer Krise versuchen, durch einen umfassenden Erstschlag (first strike) die Kernwaffenarsenale der anderen Seite weitgehend auszuschalten und deren verbleibende Offensivwaffen durch Abwehrsysteme zu neutralisieren. ${ }^{27}$ Auch auf die Risiken eines unbegrenzten Rüstungswettlaufs zwischen Offensiv- und Defensivsystemen wurde hingewiesen. ${ }^{28}$

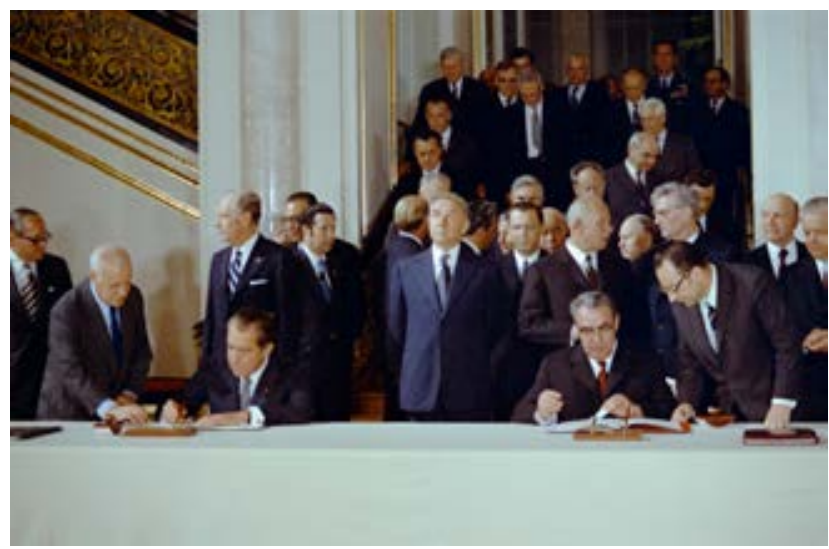

Die Unterzeichnung des SALT I- und des ABM Abkommens im Mai 1972 in Moskau

Tatsächlich setzten entsprechende Gespräche zwischen Washington und Moskau im Jahr 1969 ein und führten neben dem SALT I-Abkommen von 1972, das die Zahl der landgestützten Interkontinentalraketen begrenzte und Raketenabwehrsysteme weitgehend verbot, $\mathrm{zu}$ einer Reihe vielfach stillschweigender Übereinkünfte bezüglich technischer Parameter und über Maßnahmen, die eine politische Kontrolle des Kernwaffeneinsatzes herstellten und die Kommunikation unter Krisenbedingungen verbesserten. Dem lag die zentrale Vorstellung zugrunde, ein Zustand beiderseitig gesicherter Zweitschlagsfähigkeit würde eine robuste Form von Stabilität erzeugen. Die Ergebnisse des strategischen Dialogs stellten allerdings die Glaubwürdigkeit der erweiterten Abschreckung auf die Probe. So entstand in der US-Regierung ein Prioritätenkonflikt zwi- schen einerseits der Notwendigkeit globaler nuklearstrategischer Stabilität und andererseits den Erfordernissen der erweiterten Abschreckung und damit der strategischen Stabilität auf europäischem Boden. Während Großbritannien und Frankreich dieses Dilemma für sich durch eigene Kernwaffenprogramme lösten, stand der Bundesrepublik Deutschland diese Option knapp 20 Jahre nach Ende des Zweiten Weltkriegs aus nachvollziehbaren Gründen nicht zur Verfügung. Da sich die USA mit der Sowjetunion schon 1968 auf die Parameter eines globalen Nichtverbreitungsregimes geeinigt hatten (Nuklearer Nichtverbreitungsvertrag von 1970), war nicht zu erwarten, dass sich mit der Zeit an diesem Zustand etwas ändern würde. Zudem engte der Nukleare Nichtverbreitungsvertrag die Möglichkeiten einer multilateralen Atomstreitmacht der NATO (MLF) erheblich ein. ${ }^{29}$ Die einzigen Chancen, die sich der Bundesrepublik sowie den anderen mittleren und kleinen NATOStaaten wie Italien, Belgien und den Niederlanden (sowie auch der Türkei) boten, waren:

- Die Mitwirkung in der Nuclear Planning Group der NATO, bei der es um das Festlegen von Prinzipien und Richtlinien für den Einsatz von US-Kernwaffen zur Verteidigung Europas ging.

- Die Vornestationierung von taktischen Kernwaffen in der Bundesrepublik Deutschland und anderen europäischen Ländern, um die USA im Fall einer Aggression des Warschauer Pakts unter Zugzwang zu setzen (nach dem Prinzip use them or loose them).

- Die nukleare Teilhabe, bei der Einheiten der Bundeswehr Zugang zu taktischen Kernwaffen (Gefechtsfeldwaffen) der USA erhielten und - nach erfolgter Freigabe durch den US-Präsidenten - auch einsetzen konnten. Die Teilhabe betraf damals Flugzeugbomben ebenso wie nukleare Artillerie oder Kurzstreckenraketen sowie die Pershing 1a, die eine Reichweite von $730 \mathrm{~km}$ hatte und auch Ziele in Polen und dem sowjetischen Kaliningrad Oblast erreichen konnte. Die Pershing 1a bedeutete die Mitwirkung deutscher Streitkräfte bei der - im Rahmen der durch die NPGRichtlinien festgelegten - nuklearen Einsatzplanung und deren eventueller Durchführung unter dem Aspekt der vorbedachten Eskalation. Für Gefechtsfeldwaffen bestanden bilaterale Übereinkommen zwischen den USA und mehreren NATO-Staaten (darunter Deutschland), die Einsätze nuklearer Gefechtsfeldwaffen unter Dual-key-Verabredungen regelten. ${ }^{30}$

26 Zuerst hat auf diese Risiken Albert Wohlstetter hingewiesen, vgl. Wohlstetter 1959.

27 Vgl. McNamara 1970, 47 ff. sowie Kaufmann 1964.

28 Vgl. Shulman 1969, Feld 1969, Bethe 1969.

29 Vgl. Lutsch 2020, Kap. II, III und IV. 30 Heymont 1966. 
- Die Risikogemeinschaft generell, die sich vor allem daraus ergab, dass US Truppen an vorderster Stelle bei der mobilen Raumverteidigung in Mitteleuropa standen und daher bei Ausbruch eines Krieges sofort angegriffen und in die Pflicht genommen würden.

Diese hier aufgezeigte Kompromisslinie geriet Ende der 70er Jahre durch die sowjetische Aufrüstung bei Mittelstreckenraketen schwer unter Druck. Ermuntert durch den Abschluss des SALT-II-Vertrags, in dem die USA auf die Entwicklung seegestützter nuklearfähiger Marschflugkörper von über $600 \mathrm{~km}$ Reichweite und bestimmter luftgestützter Marschflugkörper gleicher Reichweite verzichteten, stationierte die Sowjetunion landgestützte Mittelstreckenraketen vom Typ SS-20 und Bomberflugzeuge, die eine nukleare Bedrohung aller europäischen Hauptstädte ermöglichten. Die sowjetische Rüstung sollte offenkundig eine Eskalationsdominanz herstellen und so jeden Versuch der NATO vereiteln, $\mathrm{zu}$ ihren Bedingungen einen von der Sowjetunion ausgelösten Krieg zu beenden. Im Kern ging es um die strategische Abkoppelung Mitteleuropas von den USA. Die Antwort darauf war der NATO-Doppelbeschluss vom Dezember 1979, der einerseits vorsah, in Mitteleuropa ab Mitte des Jahrzehnts amerikanische Mittelstreckenraketen und Marschflugkörper aufzustellen, die auch sowjetisches Territorium treffen konnten, und andererseits ein Rüstungskontrollangebot enthielt. ${ }^{31}$ Der politische Kampf um die Umsetzung der Rüstungsabsichten des NATO-Beschlusses spaltete vor allem in Deutschland Regierung und Öffentlichkeit, bis im Oktober 1983 der Bundestag mit deutlicher Mehrheit die Aufstellung der US-Systeme auch auf deutschem Boden befürwortete.

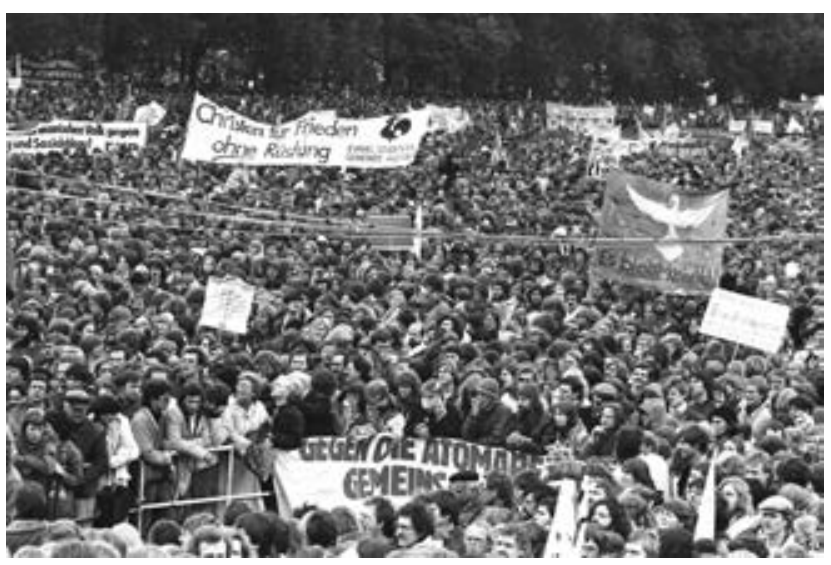

Proteste gegen den NATO-Doppelbeschluss im Bonner Hofgarten im Oktober 1981

31 Die Entwicklung, die zum NATO-Doppelbeschluss führte, ist sehr gut dargestellt bei Spohr 2016, 18-297.
Während in den 80er Jahren im Zuge der Kritik am NATODoppelbeschluss vom Dezember 1979 das irreführende Bild eines mehr oder weniger unmittelbar bevorstehenden Kernwaffenkriegs in Europa gezeichnet wurde, stellte sich im Hintergrund aus westlicher Sicht eine nachhaltige Entspannung der militärischen Lage ein. So übernahmen die US-Streitkräfte Anfang der 80er Jahre mit der AirLandBattle-Doktrin und dem Follow-on-Forces-Attack-Konzept (FOFA) Verfahren der kombinierten Land-Luft-Kriegführung. Diese stärkten nicht nur erheblich die Vorneverteidigung gegen die angreifende erste strategische Staffel des Warschauer Pakts, sondern erlaubten es auch, nachrückende Kräfte der zweiten und dritten Staffel beim Vormarsch aus dem rückwärtigen Bereich des Aufmarschgebiets des Warschauer Pakts frühzeitig anzugreifen und damit unschädlich zu machen (extended battlefield). ${ }^{32}$ Auch die NATO hatte das Follow-On-Forces-Konzept übernommen. Mit ihm wollte man auch die nachrückende zweite und dritte strategische Staffel während ihres Vormarsches durch Polen und die DDR durch Luftangriffe (in Großbritannien stationierte F-111 Langstreckenbombern und deutsche Tornado-Kampfflugzeugen mit panzerbrechenden Streubomben) so weit in ihrer Kampfkraft schwächen, dass die gegnerischen Panzerverbände bei Eintreffen an der Front keine operativen Durchbrüche mehr erzwingen könnten.

Diese von der deutschen Friedensbewegung und auch großen Teilen der SPD massiv bekämpften Innovationen änderten das Kräfteverhältnis entscheidend, da jetzt Vorteile der Verteidigung offenkundig wurden. ${ }^{33}$ Die sowjetischen Streitkräfte hatten ähnliche Modernisierungspläne wie die USA, ${ }^{34}$ konnten zu dem Zeitpunkt technologisch jedoch nicht mehr gleichziehen, denn die Defizite der sozialistischen Wirtschaft und der kommunistischen Herrschaft traten immer deutlicher zutage. ${ }^{35}$ Auch wegen der Modernisierung der Bundeswehr infolge der Heeresstrukturreform IV sahen die Planer auf Seiten des Warschauer Pakts immer geringere Chancen für die Umsetzung einer Planung, die auf die operative Offensive setzte. ${ }^{36}$ Mitte der

32 DeLauer 1986.

33 Vgl. hierzu aus der Sicht eines früheren Armeegenerals der Roten Armee Garejew 1996, 32, 37 ff; siehe auch Fitzgerald 1987.

34 Vgl. das Interview mit dem damaligen Chef des sowjetischen Generalstabs, Marschall N.W. Ogarkow in der Militärzeitschrift Krasnaja Swesda vom 9.5.1984.

35 Diese Defizite wurden in der akademischen Literatur seinerzeit wahrgenommen, aber kaum jemand konnte sich vorstellen, dass dieses Problem zu einer grundsätzlichen Umorientierung der sowjetischen Militärpolitik führen werde, vgl. etwa Becker 1982 oder Fewtrell 1983.

36 Lautsch 2013, 121. 
80er Jahre nahm die Sowjetunion angesichts der veränderten Lage Abstand von einer operativen Planung, die auf eine großangelegte Offensive gegen Westdeutschland und Westeuropa zielte und nuklearen Gefechtsfeldwaffen eine wesentliche Rolle zuwies (obwohl auch eine rein konventionelle Option bestand). Dieser Wandel erfolgte interessanterweise Anfang 1985, also noch vor dem Amtsantritt Michail Gorbatschows, und war ein Indiz, dass das sowjetische Militär die Aussichtslosigkeit eines umfassenden, mit Nuklearwaffen untermauerten Offensivkrieges in Mitteleuropa - wenngleich spät - verstanden hatte. Gleichzeitig stellte der Warschauer Pakt seine operativen Militärplanungen von der Offensive auf eine bewegliche Verteidigung um, die jener der NATO ähnelte und den Schluss zuließ, dass die NATO-Strategie der flexiblen Verteidigung einem im Großen und Ganzen klugen politischen Kalkül gefolgt war. Beide Veränderungen vollzogen sich stillschweigend und wurden von westlichen Beobachtern erst mit einiger Verspätung wahrgenommen. ${ }^{37}$

Im Mai 1987 verabschiedete sich der Warschauer Pakt auch offiziell von seiner offensiven Militärdoktrin und der mit ihr verbundenen Kriegsplanung. ${ }^{38}$ Im Dezember 1987 vereinbarten die USA und die Sowjetunion den Verzicht auf alle landgestützten Mittelstreckenraketen (ballistische oder Marschflugkörper) mit Reichweiten von 500 bis $5.500 \mathrm{~km}$ (INF-Vertrag). ${ }^{39}$ Nur zwei Jahre später war der Ost-West-Konflikt beendet, und der Zwei-plus-Vier-Vertrag sowie der Vertrag über Konventionelle Streitkräfte in Europa (KSE) läuteten den Abzug der Roten Armee aus Deutschland und den damaligen Mitgliedstaaten des Warschauer Pakts ein. Mit dem KSE-Vertrag wurden zudem numerische Parität bei den wichtigsten Waffensystemkategorien der Land- und Luftstreitkräfte sowie das Prinzip der Nichtzulässigkeit von Invasionsfähigkeit beschlossen..$^{40}$ In der Charta von Paris für ein neues Europa (November 1990) gestand die Sowjetunion bzw. später Russland den ehemaligen Warschauer-Pakt-Verbündeten und sogar ehemaligen Sowjetrepubliken (wie die Ukraine, Georgien und die baltischen Staaten) uneingeschränkte Souveränität und damit das Recht auf freie Bündniswahl $\mathrm{zu}^{41} \mathrm{Im}$ Vertrauen auf diese Garantien gab die Ukraine

37 Vgl. Lautsch 2013 (138ff), der als Offizier der NVA an den betreffenden Planungen auf Seiten der NVA beteiligt war.

38 Erklärung des Politischen Beratenden Ausschusses des Warschauer Paktes über Militärdoktrinen vom 29. Mai 1987, in: Krause/ Magiera-Krause 1992, 171-173.

39 Zum Text des Vertrags und aller dazu gehörigen Protokolle vgl. Krause/Magiera-Krause 1992, 811-859.

40 Vgl. die Dokumentation und Kommentierung bei Hartmann/Heydrich/Meyer-Landrut 1994.

41 Text der Charta von Paris in Krause/Magiera-Krause 1997, 113-121. sogar ihren aus sowjetischen Beständen geerbten Besitz an Nuklearwaffen auf. Die Probleme mit der Glaubwürdigkeit der erweiterten Abschreckung hatten sich damit erledigt. Sowohl die USA als auch Russland reduzierten ihre nuklearstrategischen Arsenale drastisch. Das US-Militär vernichtete nach und nach bis auf einen kleinen Bestand von etwa 500 B-61 Flugzeugbomben ihr gesamtes Arsenal an taktischen Kernwaffen, während Russland sein taktisches Arsenal auf etwa 2.000 Waffen reduzierte, sich aber im Gegensatz zu den USA keine Beschränkungen qualitativer Art auferlegte.

\section{Die heutige Bedrohungslage}

Heute, mehr als 30 Jahre nach Beendigung des Ost-WestKonflikts, sieht die militärische Lage anders aus. Russland unter Präsident Wladimir Putin will wieder Großmacht sein und versteht sich als strategischer Gegner des Westens - auch im militärischen Bereich..$^{42}$ Strategisch gesehen ist die neue Bedrohung defensiver Natur insofern, als die russische Führung ihre innenpolitische Position durch Demokratie, Freiheit und Stärke der westlichen Welt angegriffen sieht. ${ }^{43}$ Aber für Russlands Nachbarn hat die russische Strategie eine offensive Stoßrichtung, die sich sowohl militärischer Instrumente als auch eines breiten Instrumentariums bedient, um die westlichen Staaten und Gesellschaften und die amerikanischen Bündnissysteme zu schwächen. ${ }^{44}$ Zunehmend in den Vordergrund rückt jedoch auch die Möglichkeit von Russland entfachter regionaler Kriege. ${ }^{45}$

Daher identifiziert die nationale Sicherheitsstrategie der US-Regierung seit 2018 Russland als einen strategischen Rivalen und potenziellen militärischen Gegner, gegen den es militärische Vorsorge zu treffen gilt. ${ }^{46}$ Diese Einschätzung findet sich seit 2014 unisono auch in den Verlautbarungen der Treffen der NATO-Außenminister und NATO-Verteidigungsminister sowie in den Kommuniqués der NATO-Gipfeltreffen der Staats- und Regierungschefs wieder. Das NATO-Gipfelkommuniqué vom Juni 2021 beschreibt klar die Gefährdung durch Russland und sieht diese vor allem darin, dass militärische Drohungen gegen Nachbarstaaten und der Einsatz von Militär zur Verfolgung russischer Interessen mit einer zunehmenden

\section{Adomeit 2021.}

43 Bruusgard 2016.

44 Vgl. u. a. Clark 2020, Giles 2016, Kanet 2017, Cohen/Radin 2019. 45 Roberts 2020.

46 Vgl. Thränert 2018. 
Risikobereitschaft einhergehen. ${ }^{47}$ Bei der Natur der russischen militärischen Bedrohung, die sich für westliche Demokratien durch das mutmaßlich defensive strategische Konzept Russlands ergibt, zeichnet sich in Kreisen der Wissenschaft und der beteiligten Stäbe in der NATO ein Konsens ab, der sich wie folgt zusammenfassen lässt:

- Russland stellt anders als die Sowjetunion und der Warschauer Pakt keine Bedrohung im Sinn einer umfassenden kontinentalen Invasion dar. Die heutigen Streitkräfte haben zwar eine erhebliche Professionalisierung und Verbesserung erfahren und sind deutlich besser aufgestellt als im ersten Jahrzehnt dieses Jahrhunderts. ${ }^{48} \mathrm{Zu}$ einer kontinentalen Invasion sind sie aber nicht fähig. Sie besitzen außer Belarus keine Bündnispartner mehr und stehen auch nicht in der Stärke von 20 Divisionen in der Mitte Europas. Die militärische Bedrohung richtet sich gegen Staaten an der Peripherie Russlands. Das betrifft im Schwarzmeerbereich die Ukraine, Georgien, Moldawien sowie Rumänien und Bulgarien, im Ostseebereich die baltischen Staaten, Polen, Finnland sowie Schweden und indirekt auch Deutschland, die Niederlande, Belgien und Dänemark. ${ }^{49}$ Sowohl gegenüber der Ukraine als auch gegenüber den baltischen Staaten führten Russlands Streitkräfte in den vergangenen zehn Jahren wiederholt Truppenaufmärsche in der Größenordnung von 100.000 Mann durch - sei es im Zuge der Truppenübung Zapad (2013, 2017, 2021) oder mit unprovozierten Aufmärschen gegen die Ukraine wie zuletzt im April 2021. Ziel dieser Drohpolitik ist es, insbesondere die Ukraine (und Georgien) zu destabilisieren und deren Beitritt zu westlichen Institutionen zu verhindern. Vielmehr sollen sie sich, ähnlich wie Belarus, mit einem Status minderer Souveränität gegenüber Russland abfinden. Gegenüber den Staaten des Ostseeraums, allesamt Mitglied der NATO und/ oder der Europäischen Union, ist die Stoßrichtung subtiler. Mit seinen Manöveraktivitäten hat Russland demonstriert, dass es in der Lage ist, die baltischen Staaten nach relativ kurzer Vorbereitungszeit zu besetzen und gegen Rückeroberungsversuche der NATO $\mathrm{zu}$ verteidigen. ${ }^{50}$ In der Literatur wird diese Option als die des fait accompli bezeichnet. Nach Herstellung

47 Vgl. Galeotti 2016, Sokolsky 2017, Johnson 2019.

48 Vgl. Lavrov 2018 sowie Hackett 2021.

49 Vgl. Forss/Kiianlinna/Inkinen/Hult 2013, Shlapak/Johnsen 2016, Fruehling/Lasconjarias 2016, Klain/Lundqvist/Sumangil/Pettersson 2019, Dick 2019, Wilk 2021, Estonian Foreign Intelligence Service 2021. 50 Zu Zapad 2013 vgl. die Beiträge in Zdanavičius/Czekaj 2015, zu Zapad 2017 vgl. Boulégue 2018 sowie Palmer 2018. einer solchen nicht rückgängig zu machenden Tatsache könnte Russland mit seiner nuklearen Escalate-todeescalate-Konzeption die NATO vor die Wahl stellen, entweder den Verlust der drei baltischen Staaten (und vielleicht Teilen Polens) hinzunehmen oder einen auf Europa begrenzten Kernwaffenkrieg zu riskieren.

- Russland stellt weiterhin eine nuklearstrategische Bedrohung für die USA dar, auch wenn die Arsenale verringert worden sind. Zwischen beiden besteht nach wie vor der Zustand gegenseitig gesicherter Zerstörungsfähigkeit. Das ist einerseits beruhigend, andererseits beeinträchtigt dieser Zustand die USA bei der Ausübung glaubwürdiger erweiterter Abschreckung.

- Russland versucht, durch militärische Interventionen westlichen Interessen entgegenzuwirken und vor allem autoritäre Regierungen zu stabilisieren, die durch Bürgerproteste ins Wanken gebracht wurden, wie etwa Syrien.

- Die von Russland ausgehenden Drohungen beschränken sich nicht auf klassische Streitkräfte, sondern ziehen auch sogenannte private Dienstleister (wie die berüchtigte Wagner-Gruppe) ein, die die schmutzige Arbeit verrichten. ${ }^{51}$ Hinzu kommen umfangreiche Waffenlieferungen in Krisengebiete. Gegenüber westlichen Gesellschaften betreibt es eine Art „politische Kriegführung“, indem Cyberangriffe in großem Maßstab durchgeführt, Wahlen durch Kampagnen in sozialen Medien beeinflusst und gesellschaftliche Gruppen gegen die Regierung und die Mehrheit aufgehetzt werden..$^{52}$ Diese „hybride Konfliktaustragung“ bereits im Frieden, gesteigert in Krise und Krieg, stützt sich auf zwei Beine. Erstens greift sie zu schwer zurechenbaren subversiven Aktivitäten unterhalb der Schwelle des offenen militärischen Konflikts. Diese verbindet sie, zweitens, mit dem Aufbau einer klassischen militärischen Drohkulisse durch konventionelle Streitkräfte, die der russischen Führung ein breites Spektrum militärischer Handlungsoptionen zur Verfügung stellen. Eine Steigerung findet die bereits jetzt massive Gefährdung nur noch in der nuklearen Escalate-to-Deescalate-Drohung. Das trifft die NATO an ihrer politisch und militärisch empfindlichsten Stelle. Schließlich ist sie ein Bündnis von dreißig souveränen Nationen mit unterschiedlichen Interessenlagen. Nach Artikel 4 muss in Konsultationen auf Grundlage eines klaren Lagebilds bei der Feststellung und Zuordnung einer Aggression durch einen Angreifer

51 Vgl. Fainberg 2017. 52 Vgl. Giles 2016, Cohen/Radin 2019, Galeotti 2019, Clark 2020. 
Einvernehmen hergestellt werden. Gleiches gilt nach Artikel 5 bei Feststellung des Bündnisfalls (Artikel 5). Im Fall Russland ginge es um einen Krieg mit einer nuklear hochgerüsteten euro-asiatischen Großmacht und damit um eine für alle Verbündeten existenzielle Frage. Am deutlichsten offenbarte sich die Wirksamkeit der von Putins Russland verfolgten hybriden Kriegführung 2014 in der völkerrechtswidrigen russischen Aggression gegen die Ukraine mit der Annexion der Krim, der Besetzung von Teilen des Donbass und der seither anhaltenden Destabilisierung der Ukraine.

Wie die heutige Lage ist auch die Frage nach dem Stellenwert von Kernwaffen in der NATO-Verteidigungsstrategie eine andere als zu Zeiten des Ost-West-Konflikts. Szenarien einer militärischen Invasion Russlands in NATO-Mitgliedstaaten im Ostseeraum räumen westlichen Kernwaffen eine zentrale Rolle als Instrument der Abschreckung ein. Ihre Funktion wäre jedoch nicht dieselbe wie zu Zeiten der Konfrontation zwischen Warschauer Pakt und NATO. Kernwaffen sind als Teil eines umfassenderen Konzepts zur Abschreckung von russischen Invasionen zu verstehen und nur unter bestimmten Bedingungen ins Spiel zu bringen.

\section{Ziele und Instrumente westlicher Abschreckungspolitik in Europa}

Ziel der Abschreckungspolitik des westlichen Bündnisses muss es sein, Russland von einer raschen Eroberung der baltischen Staaten (und Polens) - dem fait accompli - abzuhalten. Das müsste eigentlich durch eine Verstärkung der konventionellen Verteidigungskräfte vor Ort geschehen. Diese müssten in der Lage sein, eine rasche und erfolgreiche Invasion zu verhindern und die Kosten einer Invasion so hoch zu treiben, dass die russische Seite sie gar nicht erst ins Auge fasst. ${ }^{53}$ Diese Option verfolgen die Mitgliedstaaten der Nordatlantischen Allianz allerdings aus politischen und militär-geographischen Überlegungen nicht. Wichtigster politischer Grund ist das Bestreben, die Bestimmungen der NATO-Russland-Akte von 1997 nicht zu verletzen, denen zufolge die NATO in den neu aufgenommenen Mitgliedstaaten keine substantiellen militärischen Kampfverbände aufstellt (d.h. weniger als eine alliierte Brigade pro Land, zusätzlich zu den jeweiligen nationa-

53 Vgl. Kramer/Binnendijk 2018, Kroenig 2018, Vershbov/Breedlove 2019, Major 2019. len Heimatschutzverbänden). In der NATO-Russland Akte verzichtet die NATO ferner auf Absichten, Planungen und Infrastrukturen für die Stationierung von Nuklearwaffen (die drei nuklearen No's). Obwohl Russland mehrfach die Regeln gebrochen hat, hält die Mehrheit der NATO-Staaten an ihnen fest. Andererseits haben sich die NATO-Staaten (darunter Deutschland) verpflichtet, die mobilen Einsatzkräfte so auszubauen, dass im Krisenfall die derzeitige Präsenz ausländischer Kräfte in den baltischen Staaten deutlich verstärkt werden kann. Allerdings wird auch das nicht genügen, um die baltischen Staaten erfolgreich verteidigen zu können. Der wesentlichste militärgeographische Grund für das Zögern der NATO ist die geringe strategische Tiefe der baltischen Staaten.

Angesichts dieser Umstände rücken Kernwaffen zwangsläufig mehr in den Fokus. Sie sollten zwei unterschiedlichen Funktionen dienen:

1. Der Androhung einer vorbedachten nuklearen Eskalation (durchaus ähnlich wie zu Zeiten der OstWest-Konfrontation), um Russland zum Abzug aus eroberten Gebieten zu bewegen. Die Zielplanung müsste von Anbeginn an Orte auf dem Territorium Russlands umfassen sowie einen oder wenige nukleare „Warnschüsse".

2. Der Abschreckung einer nuklear unterfütterten russischen Eskalationsdominanz bzw. der Verhinderung einer russischen nuklearen De-Eskalationsstrategie. Derzeit muss man davon ausgehen, dass russische Kriegspläne vorsehen, nach der raschen Eroberung der baltischen Staaten durch konventionelle Truppen Gegenmaßnahmen der europäischen NATO-Staaten abzuschrecken, indem man nukleare Schläge in der Tiefe des Raumes androht und diese Drohung unterstreicht durch präzise, mit konventioneller Sprengmunition ausgeführte Schläge gegen militärische Ziele in Deutschland oder im Gebiet der BeNeLux-Länder. ${ }^{54}$

Die erste Funktion sollte nicht aufgegeben werden, dürfte derzeit aus politischen Gründen und aufgrund mangelnder Fähigkeiten der westlichen Allianz aber nur noch partiell realistisch sein. Eine derartige Strategie wäre in vielen Ländern Europas - zumindest in Deutschland - politisch schwer durchsetzbar. ${ }^{55}$ Und sie könnte sich nicht umsetzen lassen, sollte sich die Biden-Administration zu der deklaratorischen Politik entscheiden, wonach es einziger Zweck amerikanischer Nuklearwaffen ist, den Einsatz von Kernwaffen gegen die USA und ihre Verbündeten

54 Kroenig 2018, Brauß/Krause 2019, Woolf 2021. 55 Schulte 2015, 124f. 
abzuschrecken (siehe unten). Zudem gäbe es zu wenige nukleare Waffensysteme mittlerer Reichweite, um eine glaubhafte Drohkulisse aufbauen zu können. Die USA haben in den 90er Jahren des vergangenen Jahrhunderts nicht nur ihre landgestützten Kernwaffenträger abgerüstet, sondern auch alle Kernwaffen auf Kriegsschiffen und nicht-strategischen U-Booten ausgemustert und vernichtet. ${ }^{56}$ Lediglich luftgestützte, frei fallende Bomben des Typs B-61 hat die Air Force beibehalten, von denen nach informierten Schätzungen zwischen 100 und 150 in Europa lagern. ${ }^{57}$ Diese müssten von Flugzeugen nahe ans Ziel gebracht werden und werden zurzeit modernisiert (B61/12). Ihr Hauptproblem wäre die russische Luftabwehr. Derzeit plant man in den USA, seegestützte TomahawkMarschflugkörper nuklearfähig auszurüsten, und in Großbritannien befinden sich seit einiger Zeit immer wieder strategische Bomber der US-Airforce, die nuklearfähig sind. Dem stehen aber in Europa immer noch mehr als 2.000 russische Nuklearwaffen mittlerer Reichweite gegenüber, die land-, see- und luftgestützt sind oder von U-Booten aus gestartet werden können. Zumeist handelt es sich bei den Trägersystemen um Marschflugkörper (teilweise mit hypersonischen Eigenschaften).$^{58}$ Doch auch ballistische Raketen sind darunter, so die Iskander-M Raketen (9M723), die mit einer Reichweite von bis zu 500 km von der russischen Exklave Kaliningrad aus Berlin, Warschau oder Kopenhagen erreichen können. Ebenfalls dazu zählt die noch nicht eingeführte, jedoch komplett entwickelte ballistische Rakete RS-26, die Ähnlichkeiten mit der SS-20 aufweist (sie ist zweistufig) bei weit größeren Reichweiten von über $5.500 \mathrm{~km} . .^{59}$ Aus Sibirien abgefeuert, ließen sich mit ihr Ziele in Europa sehr präzise abdecken. Gegenüber diesem Potential wirken die in Europa gelagerten nichtstrategischen Kernwaffen der USA - selbst wenn man Großbritanniens und Frankreichs einberechnen würde bescheiden. Angesichts der Größenordnung der hier befindlichen Kernwaffen lässt sich eine Strategie der vorbedachten Eskalation kaum überzeugend ins Auge fassen.

In den vergangenen zehn Jahren hat Russland seine nukleare Drohkulisse gegenüber Europa ohne westliche

56 Kristensen 2015, 141, Woolf 2021, $23 \mathrm{f}$.

57 Kristensen/Korda 2021, die Autoren gehen davon aus, dass derzeit nur 100 B-61 Bomben in Europa stationiert sind, vor wenigen Jahren seien es 150 gewesen. Die Verringerung lässt sich weitgehend auf den Abzug amerikanischer Kernwaffen aus einem Lager in der Türkei erklären. Zudem ergeben sich Schwankungen aufgrund des derzeitigen Austauschs von alten gegen modernisierte B-61 Bomben.

$58 \mathrm{Zu}$ den Schätzungen über die russischen nicht-strategischen Kernwaffen vgl. Woolf 2021, $30 \mathrm{f}$.

59 Hinz 2019, 171.
„Provokation“ massiv ausgebaut und verfeinert. ${ }^{60}$ Diese „Investition“ macht nur Sinn als Instrument der Einschüchterung und nuklearen Eskalationsdominanz in regionalen Kriegen. Im englischen Sprachraum nennt man das nukleare Deeskalation, gemeint ist die neue russische Escalate-to-Deescalate-Konzeption. Indiz für die enorme russische Aufrüstung im Bereich der nuklearen Mittelstreckenraketen sind auch die immens ausgeweiteten Lagerkapazitäten für Kernwaffen auf der Kola-Halbinsel und die Wiederherstellung von Kernwaffenlagern in der russischen Exklave Kaliningrad. ${ }^{61}$

Deswegen gewinnt die zweite Funktion westlicher Kernwaffen an Bedeutung, das Verhindern einer russischen Eskalationsdominanz bei Kernwaffen in Europa. Die russischen Rüstungsanstrengungen lassen das mehr als geboten erscheinen. Größtes Hindernis ist, dass man in Berlin und anderen europäischen Hauptstädten die nukleare russische Mittelstreckenrüstung politisch nicht oder kaum wahrnimmt. Ende der 70er Jahre, als die damalige Sowjetunion die ersten SS-20 Mittelstreckenraketen dislozierte, war es Bundeskanzler Helmut Schmidt, der auf die damit verbundenen Gefahren hinwies und eine Kombination aus konditionierter Nachrüstung und Rüstungskontrolle vorschlug. Heute gelangt nichts dergleichen an die Öffentlichkeit. Dennoch müssen konzeptionelle Überlegungen zur künftigen Rolle von Kernwaffen der NATO von der beschriebenen Bedrohungslage ausgehen. Dabei ist zu bedenken, dass man der „eurostrategischen“ nuklearen Bedrohung seitens Russland nicht nur Mittel der nuklearen Vergeltung, sondern auch defensive Instrumente sowie nichtnukleare offensive Mittel entgegensetzen sollte.

Die Abschreckung einer russischen Escalate-to-De-escalate-Strategie bedarf mindestens vier unterschiedlicher Säulen:

1. Eine europaweit wirkende, erweiterte Luftabwehr, die in der Lage ist, russische Marschflugkörper zu identifizieren und rechtzeitig abzufangen: Auch sollte dieses Luftabwehrsystem eine Raketenabwehrkomponente besitzen, die Kurz- und Mittelstreckenraketen abzufangen vermag. ${ }^{62}$ Hier besteht ein großer Nachholbedarf, weil taktische Luftabwehr in den vergangenen Jahrzehnten sträflich vernachlässigt und die 2010 eingeführte Raketenabwehr der NATO dezidiert so ausgerichtet wurde, dass sie sich nicht gegen russische Raketen einsetzen lässt. Dieser von der NATO er-

60 Woolf 2021, 31.

61 Kristensen 2018, Nilsen 2018.

62 Raketenabwehr und Abschreckung schließen sich nicht aus, vgl. Thränert/Kartchner 2015. 
kannte Bedarf soll in den kommenden Jahren gedeckt werden.

2. Nicht-nukleare Einsatzmittel, die see- und landgestützte Trägermittel russischer Kernwaffen einem hohen Risiko aussetzen können: Das betrifft insbesondere die Ortung und Bekämpfung russischer Kriegsschiffe und U-Boote, die mit nuklear bestückten Mittelstreckenwaffen ausgestattet sind. Aber auch fest installierte Einrichtungen in der russischen Exklave Kaliningrad, die der Vorbereitung und Durchführung von Raketenangriffen dienen, sollten bekämpft werden können.

3. Nicht-nukleare Mittel der Vergeltung: Hierzu könnten Cyber-Attacken großen Stils gehören und andere Formen großflächiger Lähmung wichtiger Institutionen und kritischer Infrastruktur in Russland.

4. Mittel nuklearer Vergeltung: Da die hier genannten Säulen allein nicht ausreichen würden, um vor einer nuklearen Bedrohung oder gar einem nuklearen Angriff Russlands abzuschrecken, bleiben nukleare Angriffsmittel unverzichtbar. Diese müssten in oder nahe Europa stationiert und so ausgestattet sein, dass sie Ziele in Russland treffen können. Nur so kann gesichert werden, dass das nukleare Risiko für Moskau bestehen bleibt und Russland sich nicht als Sanktuarium betrachten kann. Die Angriffsmittel müssten erkennbar nicht-strategischer Natur in dem Sinn sein, dass sie Russlands Zweitschlagfähigkeit nicht infrage stellen, weil es oberstes Ziel bleibt, einen strategischen Schlagabtausch mit Kernwaffen zu vermeiden. Für die Europäer hätten diese Mittel gleichwohl strategische Bedeutung.

Damit fällt der Blick auf die möglichen Vergeltungswaffen in Europa. Hier ist die Bilanz derzeit - wie oben schon bemerkt - relativ bescheiden. Es gibt zwischen 100 und 150 taktische Kernwaffen (B-61), die durch Flugzeuge ausgebracht werden können und unter amerikanischer Aufsicht in mehreren europäischen Ländern lagern, so auch in Deutschland. Sie lassen sich durch amerikanische Flugzeuge oder Flugzeuge der europäischen Alliierten zum Einsatz bringen (Dual Capable Aircraft - DCA). Die B-61-Kernwaffen werden derzeit modernisiert. Sie allein würden aufgrund ihrer begrenzten Anzahl und nicht zuletzt wegen ihrer fraglichen Eindringwahrscheinlichkeit in den russischen Raum ein Vergeltungspotential darstellen, das möglicherweise in Russland nicht den erwünschten Abschreckungseffekt hätte. Daher hat die amerikanische Seite drei weitere Optionen ins Gespräch gebracht:

1. Die Anschaffung eines nuklear bewaffneten, seegestützten Tomahawk-Flugkörpers der US-Navy, dem es gelänge, aus relativ sicheren maritimen Positionen heraus einzelne Ziele in Russland mit hoher Präzision anzugreifen.

2. Die Ausrüstung von strategischen Unterseebooten der US-Navy mit Sprengkörpern niedriger Sprengkraft (low-yield-warheads), die ebenfalls aus sicheren maritimen Positionen heraus gezielte Angriffe auf russisches Territorium ermöglichen würden. Hier wurde auch vorgeschlagen, einen Teil der U-Boot-gestützten strategischen Nuklearwaffen für europäische contingencies bereitzustellen. ${ }^{63}$

3. Die Verlegung einzelner strategischer Bomber nach Europa (z. B. Großbritannien), die in der Lage wären, nuklear bewaffnete Marschflugkörper mit geringer Sprengkraft abzufeuern. Derartige Verlegungen ließen sich in Krisenzeiten vornehmen.

Diese Optionen würden tatsächlich ein einigermaßen klares Gleichgewicht „eurostrategischer“ Natur schaffen, aber man sollte Frankreichs und Großbritanniens Kernwaffen nicht außer Acht lassen. Frankreich beteiligt sich jedoch weiterhin nicht an der nuklearen Planung der NATO, sondern beharrt auf strikter nationaler Unabhängigkeit. Seine Waffen sind für Russland trotzdem ein Faktor, mit dem es im ungünstigen Fall rechnen muss.

Allerdings befindet sich der seegestützte, nuklear bewaffnete Tomahawk-Flugkörper derzeit noch in der Planungsphase, und es ist nicht einmal sicher, ob dieses Projekt tatsächlich realisiert wird. Und gegen die von strategischen U-Booten oder strategischen Bombern abzufeuernden Einzelschläge lässt sich einwenden, dass auf russischer Seite der Eindruck entstehen könnte, ein umfassender nuklearstrategischer Angriff seitens der USA stünde unmittelbar bevor. Das könnte einen umfassenden Einsatz der russischen strategischen Angriffswaffen auslösen. Angesichts der heutigen sehr präzisen Aufklärungsmöglichkeiten auf diesem Gebiet (und der Möglichkeiten der Kommunikation mit dem Gegner) dürfte diese Gefahr aber als recht gering einzuschätzen sein. Ein weiteres Argument gegen low-yield-warheads lautet, sie würden die Bereitschaft, Kernwaffen einzusetzen, erhöhen. Dieser seit den 60er Jahren immer wieder von Rüstungskritikern vorgebrachte Einwand hat sich bislang nicht bewahrheitet. Die nukleare Schwelle bleibt bestehen, egal wie hoch die Sprengkraft ist. Im Fall einer militärischen Konfrontation in Europa könnten Kernwaffen niedriger Sprengkraft eher entscheidend zu ihrem Nichteinsatz beitragen.

Eine weitere, in der akademischen Diskussion aufgeworfene Option, wäre, die im Rahmen der nuklearen Teil-

63 Larsen 2015, 61. 
habe einzusetzenden Flugzeuge nicht mit B-61/12-Bomben auszustatten, sondern mit Abstandswaffen (also einem Marschflugkörper mittlerer Reichweite). Das könnte die Eindringwahrscheinlichkeit deutlich verbessern und die Risiken für die Flugzeuge verringern. ${ }^{64}$ Diese Option spielt indes derzeit keine Rolle in den politischen Beratungen.

\section{Die Diskussion über Kernwaffen und Allianzbeziehungen in den USA}

Da es beim Thema Kernwaffen in der atlantischen Allianz (vor allem) um amerikanische Kernwaffen geht und die USA aus vielen anderen Gründen die führende Rolle innerhalb der NATO spielen, kommt man nicht umhin, einen Blick auf die derzeitige Diskussion in den USA zu werfen. Diese ist vielschichtiger als in Europa und zeichnet sich dadurch aus, dass man die anstehenden Probleme und die militärischen und politischen Optionen sehr viel klarer anspricht als in Deutschland und den meisten europäischen Staaten - mit Ausnahme Großbritanniens, Frankreichs und der baltischen Staaten. Drei Themen dominieren die relevanten amerikanischen Debatten:

1. Der fundamentale Wandel der internationalen Beziehungen, insbesondere die konfrontative Politik unter $X i$ Jinping: China fordert die USA politisch und zunehmend auch militärisch heraus und wird als peer competitor begriffen. Die militärischen Herausforderungen Seitens China im ostasiatischen Raum stellen für die USA heute das primäre Feld einer internationalen Konfrontation dar, die sowohl politische, wirtschaftliche, technologische und gesellschaftspolitische als auch militärische Komponenten hat. ${ }^{65}$

2. Zunehmende Ungeduld gegenüber den europäischen Verbündeten (mit Ausnahme Großbritanniens): Die meisten Partner in Europa sehen die strategische und vor allem militärische Herausforderung durch China, Russland und auch den Iran mit großer Gelassenheit und scheuen sich, auf militärische Debatten jenseits interner NATO-Diskussionen einzugehen. Letzteres trifft vor allem auf die bisherige Bundesregierung unter Kanzlerin Merkel zu. Die Ungeduld der USA war besonders während der Amtszeit von Präsident Donald Trump zu spüren und führte zu einer massiven Abkühlung der Beziehungen und zur Gefahr des

64 Kroenig 2018, 336. 65 Vgl. Colby 2021.
Austritts aus der Allianz. Inzwischen bemüht sich die Biden-Administration um eine Verbesserung der Beziehungen, insbesondere zur deutschen Bundesregierung. Die bisherigen Reaktionen aus Berlin und auch die Ergebnisse des Besuchs der Bundeskanzlerin in Washington Mitte Juli 2021 lassen aber nicht erkennen, dass man die amerikanische Besorgnis wirklich versteht. Daher kann die jetzige freundliche Einstellung in Washington wieder kippen. ${ }^{66}$ Das strategische Problem der NATO - die zunehmende Orientierung der USA nach Ostasien und Deutschlands und Frankreichs weitgehende Gelassenheit gegenüber der militärischen Bedrohung Europas durch Russland - wird die Abschreckungsdiskussion in den kommenden Jahren deutlich belasten. ${ }^{67}$

3. Die Notwendigkeit einer grundlegenden Modernisierung der amerikanischen nuklearstrategischen Arsenale: Diese Modernisierung gilt seit Verabschiedung des Berichts einer überparteilichen Kommission im Jahr 2016 als grundsätzlich vereinbart. Sie wird sich über mehr als zwei Dekaden erstrecken und beträchtliche finanzielle Ressourcen verbrauchen. Nach drei Jahrzehnten des Nichtstuns ist sie notwendig geworden, um die Fähigkeiten der USA im Bereich der strategischen Rüstung beizubehalten. Wichtige neue Fähigkeiten sind nicht beabsichtigt, ebenso wenig ein umfassender Ausbau eines Raketenabwehrsystems. ${ }^{68}$ Die Umsetzung dieser Modernisierungspläne führt aber immer wieder zu einer offenen Debatte über Sinn und Logik von Kernwaffen und Abschreckung, da dem Kongress bei den Entscheidungen über die Finanzierung der Teilprogramme eine zentrale Rolle zukommt. Daher wurden und werden immer wieder mehr oder weniger wesentliche Bereiche des Programms infrage gestellt. In diesen Auseinandersetzungen gewinnen Stimmen an Gewicht, die eine Modernisierung grundsätzlich anzweifeln oder Rüstungskontrolle im nuklearstrategischen Bereich zuungunsten einer erweiterten Abschreckung ausdehnen wollen. Vor allem die Argumente aus dem Lager der liberalen Rüstungskontrollbefürworter finden die Unterstützung von linken Demokraten und ihren Niederschlag auch in der BidenAdministration.

$66 \mathrm{Zu}$ den Bedingungen, unter denen eine transatlantische Annäherung erfolgreich sein könnte, vgl. Krause 2021a.

67 Kamp 2019, Rühle 2020.

$68 \mathrm{Zu}$ den Hintergründen vgl. Roberts 2015 sowie Woolf 2018. 


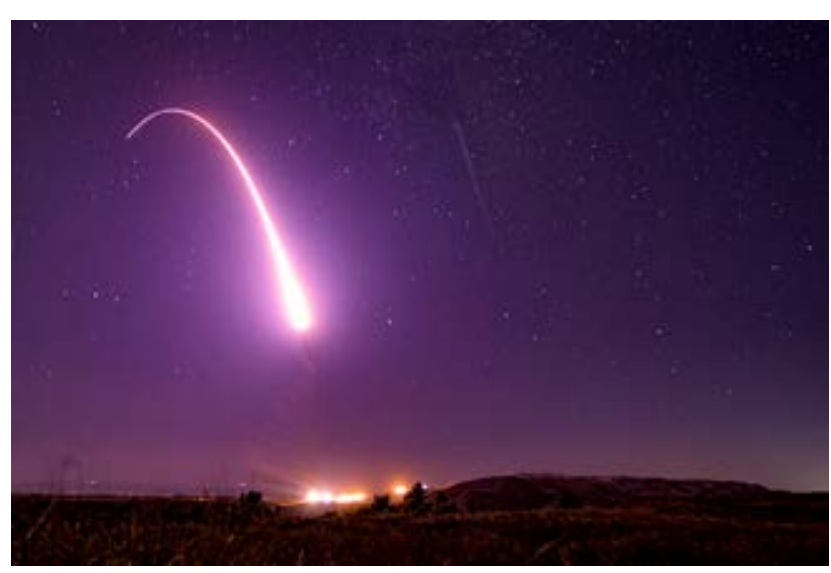

Test einer amerikanischen Minuteman-ICBM im Jahr 2019

In dieser Situation könnten sich aufgrund knapper Finanzen und Verärgerung über europäische Verbündete (insbesondere Deutschland) zwischen eher verteidigungsorientierten Republikanern und Demokraten sowie linken Demokraten Koalitionen bilden und der Spielraum für Reaktionen auf die russische Rüstung im Bereich der eurostrategischen Kernwaffen erheblich verengt werden. Während verteidigungspolitisch engagierte Republikaner immer offener fordern, vor allem Deutschland stärker in die Pflicht zu nehmen, verlangen liberale Rüstungskontrollvertreter den vollständigen Verzicht auf nichtstrategische Kernwaffen. Die Existenz amerikanischer strategischer Potenziale würde zur Abschreckung Russlands genügen, alles andere die Gefahr eines unbeabsichtigten Krieges erhöhen. ${ }^{69}$ Wenngleich derart radikale Forderungen nicht unbedingt große Aussicht auf Erfolg haben, könnten kleinere Projekte scheitern, so etwa die Beschaffung einer seegestützten nuklearen Variante des Tomahawk-Flugkörpers.

Ganz entscheidend ist die Frage, ob amerikanische Kernwaffen einzig auf den Zweck verpflichtet werden sollten, vom Kernwaffeneinsatz gegen die USA und ihre Alliierten abzuschrecken (sole purpose). ${ }^{70}$ Gemäß dieser vor allem von Rüstungskontrollanhängern geforderten deklaratorischen Politik würden die USA keine Kernwaffen mehr einsetzen (oder deren Einsatz androhen), wenn Russland oder China in einer konventionell durchgeführten militärischen Operation einen Verbündeten der USA in Europa oder Asien besetzen. Präsident Joseph Biden hat

69 Siehe Fetter/Wolfsthal 218; Perry/Colinna 2020, zur Kritik siehe Roberts 2021b.

70 Vgl. Davenport/Kimball/Reif 2021, Perkovich/Vaddi 2021; s. a. Steven Pifer: Nuclear Weapons. It's Time for Sole Purpose, The National Interest, 15.9.2020; zur Kritik siehe Roberts 2021b. bereits früher seine Sympathie für diese deklaratorische Politik erkennen lassen, sodass sie durchaus Realität werden kann. Kritiker weisen zu Recht darauf hin, dass man mit der vagen Hoffnung auf rüstungskontrollpolitische Erfolge ein wichtiges Element der amerikanischen Sicherheitsgarantie für die Verbündeten in Europa und Asien verspielen würde. ${ }^{71}$ Die Europäer - speziell die Bundesregierung - müssen mit dieser Eventualität leben. Und sie sollten keine Signale senden, die Konstellationen begünstigen, bei denen das Optionenfenster auf amerikanischer Seite sich schließt. Das gilt auch und gerade für die neue deutsche Bundesregierung. Die deutsche Debatte über nukleare Teilhabe fällt hier ins Gewicht.

\section{Die nukleare Teilhabe Deutschlands}

Zentral bei der Abfassung eines neuen strategischen NATO-Konzepts wird die Frage sein, inwieweit nach Ansicht der europäischen Verbündeten und insbesondere der deutschen Regierung die nukleare Teilhabe der NATO fortgesetzt, auf weitere Mitgliedstaaten ausgeweitet oder aber ganz aufgegeben wird. Es sind zumeist linke, sozialdemokratische und grüne Parteien, die Forderungen nach einem Abzug der amerikanischen B-61-Bomben und einer Beendigung der nuklearen Teilhabe aufstellen. In Deutschland sind sie weitgehend dieser Position verpflichtet, obwohl die SPD in der Koalitionsvereinbarung von 2018 anderes zugesagt hatte. Die Hauptargumente der Befürworter einer Abschaffung der nuklearen Teilhabe lauten:

- Diese Waffen seien militärisch nutzlos geworden.

- Die nukleare Teilhabe bewirke ohnehin keine echte Mitbestimmung beim Einsatz von Kernwaffen, weil letztendlich der amerikanische Präsident alles genehmigen müsse.

- Der Abzug der Nuklearwaffen würde ein wichtiges rüstungskontrollpolitisches Signal bedeuten. ${ }^{72}$

71 Roberts 2021a.

72 Vgl. Ralf Mützenich: Das Eskalationsrisiko ist unüberschaubar geworden, Interview mit Hans Monath, Der Tagesspiegel, 4.5.2020; ders.: Deutschland und die nukleare Teilhabe. Plädoyer für eine notwendige und ehrliche sicherheitspolitische Debatte, IPG-Journal, 7.5.2020; https://www.ipg-journal.de/rubriken/aussen-und-sicherheitspolitik/ artikel/deutschland-und-die-nukleare-teilhabe-4342/ sowie Mützenich 2020 und Fuhrhop/Kühn/Meier 2020. Siehe auch ,Baerbock fordert Abzug der US-Atomwaffen aus Deutschland‘, Epoch Times, 28.1.2021; www.epochtimes.de/politik/deutschland/baerbockfordert-abzug-der-us-atomwaffen-aus-deutschland-a3435599.html. 
Gegenüber dem ersten Argument haben die bisherigen Ausführungen deutlich gemacht, dass eine westliche Abschreckungsstrategie angesichts der Bedrohungslage und sehr massiven russischen Aufrüstung bei nuklearen Mittelstreckenwaffen außer auf strategische Offensivwaffen einzig noch auf B-61-Bomben der USA aufbauen kann - es sei denn, die Kritiker dieser Bombe wollen eine Debatte über neue eurostrategische Kernwaffensysteme beginnen.

Das zweite Argument ist unzutreffend. Nukleare Teilhabe ist heute anders gelagert als zur Zeit des Ost-WestKonflikts, in der es neben der vorbedachten nuklearen Eskalation hauptsächlich um die gemeinsame Handhabung von Gefechtsfeldwaffen unterschiedlicher Art ging und wo sich eine Vielzahl von Konsultationsmechanismen multilateraler und bilateraler Natur überlagerten. In der heutigen Praxis der NATO spielen Gefechtsfeldwaffen keine Rolle mehr. Nukleare Teilhabe erstreckt sich auf ein kleines Spektrum von Waffensystemen, eigentlich nur noch auf die B-61 Bomben, und soll primär der Abschreckung gegen eine nukleare Strategie Russlands dienen, um eine konventionell vorgetragene Invasion zu beenden. Im Einzelnen heißt nukleare Teilhabe heute, dass

- in der Nuklearen Planungsgruppe der NATO (NPG) Prinzipien und Verfahren der Einsatzplanung festgelegt werden,

- die NPG in Krisenzeiten Entscheidungsgremium über die Verwendung und Zielplanung der amerikanischen Nuklearwaffen ist, in dem alle Teilnehmer ein Vetorecht besitzen (also nicht nur der amerikanische Präsident),

- die Mitwirkung deutscher und anderer europäischer Staaten und die ständige Übung von Einsätzen und Verfahren eine Dichte der Kooperation bewirken, die Versuchen Russlands entgegenwirken soll, Europäer und Amerikaner in der nuklearen Frage zu entzweien. ${ }^{73}$

Hinzu kommt ein vor allem für Deutschland wichtiger Punkt, der in der hiesigen politischen Diskussion noch keine Rolle spielt, obwohl er eigentlich der entscheidende ist. Wie in der Bedrohungsanalyse dargelegt, ist Deutschland Hauptzielgebiet jener Mittelstreckenraketen, die Russland in den vergangenen zehn Jahren teilweise unter Verstoß gegen den INF-Vertrag aufgestellt hat. Einem immer wieder $\mathrm{zu}$ begegnenden Missverständnis zufolge

Sowie ,Nukleare Teilhabe ist ultimative Unsicherheitsgarantie‘, Pressemitteilung von Sevim Dagdelen (Die Linke), 28.4.2020; https:// www.linksfraktion.de/presse/pressemitteilungen/detail/nukleareteilhabe-ist-ultimative-unsicherheitsgarantie/.

73 Roberts 2021a und Roberts 2021b. ist Deutschland heute von Kernwaffen nicht bedroht, weil es nicht mehr (wie zu Zeiten des Ost-West-Konflikts) Gefechtsfeld eines Kriegs darstellt. Zwar dürfte Deutschland tatsächlich kein Gefechtsfeld mehr sein und muss daher keine Zerstörung durch Gefechtsfeldwaffen befürchten. Gefährlich sind heute aber nicht die Gefechtsfeldwaffen. Es sind die Raketen (ballistische wie Marschflugkörper) mittlerer Reichweite, die auf Deutschland, die Niederlande und Belgien zielen, weil hier die logistische Infrastruktur der NATO-Verteidigung liegt und zudem alle drei Regierungen als von Drohpolitik leicht beeinflussbar gelten. Deutschland ist der rückwärtige Raum einer NATO-Verteidigung osteuropäischer und baltischer Mitgliedstaaten und als solcher im Visier russischer Kriegsplanungen sowohl mit konventionellen wie nuklearen Einsatzmitteln. Unter diesen Bedingungen käme ein Verzicht auf nukleare Teilhabe politisch einer Kapitulation gleich.

Ein Ausstieg Deutschlands aus der nuklearen Teilhabe kann überdies dazu führen, dass andere NATO-Staaten nur allzu gern an Deutschlands Stelle treten, allen voran Polen. Dieser Schritt würde Polens Rolle bündnispolitisch aufwerten und könnte dazu beitragen, dass sich der Kurs der NATO gegenüber Russland verschärft.

Das dritte Argument der Kritiker - die Eignung eines Rückzugs der Kernwaffen aus Europa als rüstungskontrollpolitisches Signal - reflektiert realitätsfremdes Wunschdenken. In der Theorie mag es durchaus vorstellbar sein, dass einseitige Schritte und entsprechende „Signale“ eine Dynamik der gegenseitigen Abrüstung und Entspannung auslösen. Doch es gibt kaum erfolgreiche Beispiele dafür. Ein rüstungskontrollpolitisches Signal gaben die USA 1991 mit dem Abzug aller taktischen Kernwaffen aus ihrem Arsenal (ausgenommen etwa 500 B-61-Bomben) und der Umsetzung und Einhaltung des INF-Vertrags. Außerdem verzichteten sie auf die nukleare Variante des Marschflugkörpers Tomahawk, der Ziele in Entfernungen von bis zu $2.500 \mathrm{~km}$ präzise bekämpfen kann. Die Sowjetunion (und später Russland) haben 1991 darauf zwar ebenfalls mit der Abrüstung ihrer Gefechtsfeldwaffen und der Verschrottung der INF-Raketen geantwortet, behielten aber mindestens 2.000 nicht-strategische Gefechtsköpfe zurück. Darüber hinaus findet seit etwa 15 Jahren eine umfassende Modernisierung russischer Mittelstreckenwaffen statt. Dazu gehören auch Marschflugkörper mit den Eigenschaften des Tomahawks, die mit nuklearen Sprengköpfen ausgestattet sind. Solange diese Modernisierung see- und luftgestützte Marschflugkörper umfasste, blieb sie sogar im Rahmen dessen, was der INF-Vertrag erlaubte (und was dessen begrenzte Schutzwirkung offenbarte). Ein klarer Vertragsbruch war dann die Entwicklung, Erprobung und Einführung des landgestützten Marschflugkörpers CCS-8. 
Es bleibt unerklärlich, nach welcher Logik angesichts dieser Vorgeschichte zu erwarten sein soll, dass Russland ein derartiges „Signal“ positiv aufnähme. Die russische Regierung hat sich bislang Verhandlungen über nichtstrategische Kernwaffen verweigert und hat wegen der großen Ungleichgewichte keinerlei Anlass, von dieser Haltung abzuweichen. Vor allem die Modernisierungen der vergangenen zehn Jahre lassen erkennen, dass mit ihnen konkrete Planungen für regionale Kriege einhergehen. ${ }^{74}$ Ein rüstungskontrollpolitisches Angebot wird daran nichts ändern, es sei denn, die westliche Allianz stellt zuvor ein militärisches Dispositiv auf, das sämtliche Erwartungen der russischen Militärplaner für erfolgreiche regionale Angriffskriege durchkreuzt. Davon ist die NATO aber noch weit entfernt.

Hingegen würde ein „Ausstieg“ Deutschlands aus der nuklearen Teilhabe der deutschen Sicherheit und der unserer kleineren Verbündeten schaden. Er würde dazu führen, dass andere Länder sich ebenfalls aus dieser Teilhabe verabschieden. Das entzöge der Allianz die Fähigkeit, „glaubhaft zu signalisieren, dass ein nuklearer Angriff auf einen Einzelnen als Angriff auf alle Bündnispartner behandelt werden würde.“75 Die europäische Sicherheitsarchitektur geriete ins Wanken und die Gefahr eines Kriegs in Europa nähme zu.

\section{Die Rolle von Rüstungskontrolle}

Das neue Strategische Konzept der NATO wird sich natürlich auch der Rüstungskontrolle widmen und damit auseinandersetzen, ob und wie sich mit Mitteln der Rüstungskontrolle die derzeitige Bedrohungssituation abmildern oder gar beseitigen lässt. Wie oben ausgeführt, stehen dafür die politischen Zeichen schlecht. Es ist nicht absehbar, dass sich an Russlands Ablehnung solcher Verhandlungen etwas ändern wird. Auch sind die USA nach den bisherigen Erfahrungen mit der russischen Vertragsuntreue gegenüber Rüstungskontrolle skeptisch. Vor allem misst man in den USA alles daran, wie und auf welche Weise die Konkurrenz mit der Volksrepublik China tangiert wird wobei Peking bislang im Übrigen keinerlei Interesse an Rüstungskontrolle hat erkennen lassen. Dennoch macht es Sinn, sich zumindest vorzustellen, wie nukleare Rüstungskontrollregelungen aussehen könnten, die sich für eine Entspannung der jetzigen Situation eignen würden.

74 Zum Konzept regionaler Kriege in den Planungen Russlands und auch Chinas vgl. Roberts 2020.

75 Roberts 2021a, 5.
Im Mittelpunkt muss stehen: Wie ließe sich verhindern, dass Russland sich ausrechnen kann, durch Androhung des Einsatzes nuklearer Offensivwaffen eine Eskalationsdominanz (oder nukleare Deeskalation) in einem von ihm ausgelösten regionalen Krieg in Europa herzustellen?

Theoretisch sind für ein Rüstungskontrollabkommen drei Modelle denkbar:

1. Ein Vertrag, der Trägersysteme für nicht-strategische Kernwaffen verbietet und deren Zerstörung vorsieht, aber Kernwaffen unberücksichtigt lässt.

2. Ein Vertrag, der nicht-strategische Kernwaffen verbietet, nicht jedoch deren Trägersysteme.

3. Ein Vertrag, der Anzahl und Qualität nicht-strategischer Kernwaffen in Europa limitiert.

Das erste Modell entspräche im Prinzip dem INF-Vertrag vom Dezember 1989, der lediglich die Verschrottung bestehender Raketensysteme unter beiderseitiger Überwachung beinhaltete und zudem einen Konsultationsmechanismus, der zur Einhaltung der Verbotsvereinbarungen diente. Diese einfache Lösung umging damals die komplexe Frage, wie gegenseitig die Vernichtung bestehender oder die Lagerung ausgemusterter Kernwaffen zu überwachen sei. Seinerzeit war das Vertrauen in die Vertragstreue der anderen Seite groß. Der INF-Vertrag bezog sich außerdem nur auf landgestützte Mittelstreckenraketen und Marschflugkörper. Für seegestützte Marschflugkörper flankierte die amerikanische Seite den Vertrag mit dem Verzicht auf Beschaffung und Stationierung von mit Kernwaffen bestückten seegestützten Marschflugkörpern (und Raketen). In der Folge besitzen die USA heute keine nuklear bewaffneten Flugkörper, die sie von Schiffen aus gegen Russland einsetzen könnten. Gleiches gilt für luftgestützte Marschflugkörper. Auch hier beschränkten sich die USA auf nuklear bewaffnete Marschflugkörper, die sich von strategischen Bombern starten ließen (AGM-86 $A L C M)$ und damit unter den START-Vertrag und dessen Obergrenzen und Verifikationsregeln fielen.

Auf sowjetischer bzw. russischer Seite verfügte man Anfang der 90er Jahre über keine den amerikanischen Tomahawks vergleichbaren Systeme, allerdings über Raketen des Typs RK-55 (NATO Code SS-10), die man von U-Booten der Akula-Klasse starten konnte und Reichweiten von bis zu $2.400 \mathrm{~km}$ aufwiesen. Hinzu kamen luftgestützte Marschflugkörper der Typen Kh-55 und Kh-55 $S M$ mit Reichweiten von 2.500 km bzw. 3.000 km. ${ }^{76}$ Über diese Waffen schaute die amerikanische Seite großzügig

76 Ein Überblick zu den nicht-strategischen russischen Marschflugkörpern mittlerer Reichweite findet sich bei Brauß/Krause 2019, 166. 
hinweg, obwohl sie erst Mitte der 80er Jahre eingeführt worden waren. Die Großzügigkeit war vertretbar, denn diese Waffensysteme machten nur Sinn als Mittel der regionalen Eskalationsdominanz nach einer umfassenden Invasionsplanung des Warschauer Pakts. Die Auflösung des Warschauer Pakts und der Sowjetunion und der 1994 abgeschlossene Rückzug der Roten Armee aus Mitteleuropa hatten diese Systeme überflüssig gemacht.

Heute, über dreißig Jahre nach Abschluss des INFVertrags und zehn Jahre nach dem Wiederaufbau einer regionalen Invasionsfähigkeit Russlands, ist der INF-Vertrag hinfällig geworden. Russland sucht wieder die Eskalationsdominanz (oder verfolgt, wie es in der englischsprachigen Literatur heißt, eine nukleare Deeskalationsstrategie), hat den Vertrag gebrochen und auf die Zurückhaltung der USA im Bereich see- und luftgestützter Mittelstreckenraketen nicht positiv reagiert, sondern vielmehr die Vertragslücken voll ausgenutzt. ${ }^{77}$ Daher ist die Annahme, mit der Wiederherstellung des INF-Vertrags ließe sich mehr Sicherheit erzeugen, völlig unrealistisch. Einen Sicherheitsgewinn durch einen Rüstungskontrollvertrag kann man nur dann erzielen, wenn man eine Nulllösung für alle nuklearfähigen Trägersysteme anstrebt. Diese ist jedoch genauso unrealistisch und schwer zu verifizieren. Nicht bloß wären Russlands massive Investitionen in die neuen Mittelstreckenwaffen völlig entwertet, ohne dass darin Moskau einen Vorteil erkennen könnte, sondern auch die USA wären zu einem derartigen Verzicht nicht bereit. Dieser wäre angesichts der chinesischen Aufrüstung bei nicht-strategischen (und mittlerweile auch strategischen) Kernwaffen nicht mehr vorstellbar. Zudem bräuchte es nach den Erfahrungen mit dem russischen Vertragsbruch ein neues Verifikationssystem, das einerseits (weil ohne Unterscheidung zwischen landgestützten auf der einen und see- sowie luftgestützten Raketen auf der anderen Seite) einfacher, andererseits weit intrusiver sein muss als bisher. Vor allem muss gesichert sein, dass Vertragsbrüche unmittelbar Gegenmaßnahmen bewirken und kein Mitglied versuchen kann, sich vor den Konsequenzen eines Vertragsbruchs der anderen Seite zu drücken (wie es die Bundesregierung gemacht hat, die seit 2014 vom russischen Vertragsbruch wusste, trotzdem auf Fortbestand der Gültigkeit des INF-Vertrags bestand).

Wenn die Option eines vollständigen Verzichts der USA und Russlands auf Trägermittel für nicht-strategische Kernwaffen (Modell 1) nicht machbar ist, dann ist Modell $2 \mathrm{zu}$ beleuchten. Dieses würde darauf hinauslaufen, dass die Trägermittel bestehen bleiben, aber sowohl die USA

77 Vgl. Brauß/Krause 2019. als auch Russland übereinkommen, sie zumindest auf dem Schauplatz Europa nicht mit nuklearen Waffen auszurüsten. Eine solche Regelung wäre vorstellbar, wenn die Staaten der westlichen Allianz ihr volles politisches Gewicht in die Schale werfen und ihr politisches Verhältnis zu Russland davon abhängig machen, dass Russland sein Streben nach nuklearer Eskalationsdominanz in Europa einstellt. Das setzt allerdings ein Maß an Einigkeit und Entschlossenheit voraus, das derzeit in weiter Ferne liegt. Größter Hemmschuh dürfte die deutsche Bundesregierung sein, die sich seit Jahren beharrlich weigert, die russische Nuklearrüstung überhaupt als Problem anzuerkennen (zumindest gilt das für die bisherige Kanzlerin und den Außenminister), geschweige denn zu einem kritischen Aspekt des Dialogs mit Moskau zu machen. Und nach dem Ausgang der Bundestagswahl vom 26. September 2021 ist mit einer Änderung dieser Haltung nicht zu rechnen.

Nimmt man einmal kontrafaktisch an, die kommende deutsche Bundesregierung fasst sich ein Herz, macht sich tatsächlich für diese Sache stark und bewirkt gemeinsam mit den USA und Großbritannien eine solche Lösung, was wäre dann mit der Umsetzung? Kernproblem wäre die Überwachung der Verpflichtung, denn die nuklearen Sprengköpfe wären ja nicht aus der Welt. Entweder man würde sie in Rahmen eines verifizierbaren Verfahrens zerlegen und die Komponenten in einer Weise aufbewahren, dass die Rekonstitution sehr lange dauert und damit der anderen Seite genügend Zeit lässt, um zu reagieren. Oder man würde die ausgemusterten Kernwaffen so lagern, dass die andere Seite ihren Verbleib jederzeit verifizieren kann - etwa durch eine Kombination aus technischen Überwachungsverfahren und periodischen Inspektionen. All das wäre machbar, doch ein Problem bleibt und verurteilt dieses Modell zum Scheitern: die Möglichkeit der Substitution der abgerüsteten oder ausgemusterten Kernwaffen durch andere oder neue Kernwaffen. Dies ließe sich nur mit einem intrusiven Inspektionsaufwand verhindern, doch selbst das garantiert nicht das erforderliche Maß an Sicherheit und würde zudem von den USA wie von Russland als zu sensitiv abgelehnt werden. Es würde die Gefahr eines raschen Ausscherens Russlands aus dem Vertrag drohen mit gravierenden sicherheitspolitischen Konsequenzen (bis hin zum Ausbruch eines Krieges). Der relative militärische und politische Gewinn eines Vertragsbruchs könnte für die russische Seite so verlockend sein, dass ein derartiges Abkommen ein Sicherheitsrisiko bedeuten würde.

Hier käme unter Umständen das dritte Modell ins Spiel. Auch dabei müsste die westliche Welt politisch Einigkeit und Entschlossenheit zeigen und demonstrieren, dass ein gedeihliches Verhältnis zu Russland nur möglich ist, wenn 
das Spiel um nukleare Eskalationsdominanz in Europa aufhört. Dieser Paradigmenwandel entspräche jenem am Ende der 80er Jahre, als die NATO - im Übrigen erst nach einem langen Hin und Her - nicht mehr das Gleichgewicht an konventionellen Kräften zum politischen Maßstab der Rüstungskontrolle machte, sondern die Fähigkeit zur großangelegten militärischen Invasion. ${ }^{78} \mathrm{Im}$ Gegensatz zu Modell 2 könnten Russland und die USA (und wegen der nuklearen Teilhabe auch die NATO) eine begrenzte Zahl von nicht-strategischen Kernwaffen in Europa belassen, die sich technisch weniger für eine aktive Eskalationsstrategie als für deren Verhinderung eignen müssten. Erreichen ließe sich dies, indem man Russland quantitativ und qualitativ das Niveau nicht-strategischer Kernwaffen in Europa zugesteht, das derzeit die NATO hat: also zwischen 100 und 200 Kernwaffen, die nur aus Bomben bestehen, die aus Flugzeugen abgeworfen werden können. Auch hier stellen sich enorme Probleme der Verifikation - und der Bereitschaft, bei Vertragsbruch die notwendigen Konsequenzen zu ziehen. Weil jedoch jede Seite einen Bestand an Kernwaffen hat und die NATO-Regularien für nukleare Teilhabe nicht angefasst werden, könnte ein russischer Vertragsbruch genügend Handlungsspielraum belassen und nicht die katastrophalen Konsequenzen haben wie bei Modell 2.

Auch Modell 3 dürfte politisch derzeit nicht praktikabel sein und könnte an vielen Ecken scheitern. Im Vergleich $\mathrm{zu}$ den beiden anderen Lösungen besitzt es immerhin eine minimale Erfolgswahrscheinlichkeit vorausgesetzt, Deutschland, die europäischen Regierungen und die USA bringen den politischen Willen und die Entschlossenheit auf und vor allem: die deutsche Rüstungskontrollpolitik lernt, wieder strategisch zu denken. ${ }^{79}$

\section{Fazit}

Das neue Strategische Konzept der NATO wird die Rolle von Kernwaffen selbstverständlich nicht so detailliert behandeln können wie hier beschrieben. Das wird auch nicht notwendig sein. Wichtig ist aber, dass die Überlegungen eine ehrliche und realistische Auseinandersetzung mit den hier skizzierten sicherheitspolitischen Herausforderungen und Bedrohungen erkennen lassen und die richtigen allgemeinen Schlüsse gezogen werden. Das ist keinesfalls eine Selbstverständlichkeit, denn aus

78 Vgl. Krause 1988.

79 Vgl. zu den Defiziten deutscher Rüstungskontrollpolitik Krause 2021b. politischen Gründen wollen die meisten Regierungen der NATO-Staaten am liebsten nicht auf Fragen der nuklearen Abschreckung angesprochen werden. ${ }^{80}$ Dies trifft ganz besonders auf die derzeitige und die künftige deutsche Bundesregierung zu. ${ }^{81}$ Das Thema „Kernwaffen und Abschreckung “ gilt als politisch nicht vermittelbar, im Zweifelsfall zieht man sich auf allgemeine, allianzintern vereinbarte Formeln zurück. Aber selbst diese werden in die deutsche Debatte nicht übertragen. ${ }^{82}$ Ansonsten prägen überwiegend Fehlinformationen und simple, populistische Annahmen über Krieg und Frieden das öffentliche und politische Klima in Deutschland, sodass eine sachliche strategische Debatte über Verteidigungspolitik und Abschreckung kaum mehr möglich ist - obwohl sie notwendig ist. ${ }^{83}$ Keine der Bundesregierungen seit Helmut Kohl hat je versucht, eine strategische Diskussion über Aufgaben und Ziele der Bundeswehr zu führen - auch nicht in Zeiten, in denen die Bundeswehr primär für Auslandseinsätze bereitstand. Strategisch wurde nie diskutiert, weshalb und in welchem Ausmaß Deutschland die Stabilisierung Afghanistans unterstützen soll und welche die Kriterien für Erfolg oder Misserfolg sind. Und die wenigen Ansätze, wenn es sie einmal gab, hielten sich an Einzelaspekten auf und blieben eher auf der Ebene der Verarbeitung von Erfahrungen. ${ }^{84}$ Kritische Bilanzen aus der Wissenschaft, die die Chancen und Aussichten von Stabilisierungsmissionen und Interventionen beleuchteten oder aufzeigten, unter welchen Bedingungen es möglich sein könnte, eine dauerhafte Befriedung oder Stabilisierung von meist durch Bürgerkriege zerrissenen Ländern zu erzielen, wurden und werden in der Politik nicht wahrgenommen. ${ }^{85}$

Ebenfalls am liebsten gar nicht ansprechen will die bisherige Bundesregierung - mit Ausnahme der Verteidigungsministerin - das Thema, dass es (1) wieder eine militärische Bedrohung seitens Russlands gibt (wenn auch direkt nur gegen Verbündete Deutschlands) und (2) Deutschland im Fadenkreuz einer in den vergangenen

\section{Vgl. Kamp 2007.}

81 Einschränkend muss ergänzt werden, dass die Verteidigungsministerin von dieser Kritik ausgenommen werden muss. Zudem hat die CDU den Mut aufgebracht, sich in ihrem Wahlprogramm 2021 für die nukleare Teilhabe auszusprechen.

82 Man vergleiche zu diesem Zweck nur die Sprache des Gipfeldokuments der NATO von Juni 2021 (dem die Bundeskanzlerin zugestimmt hat) zur russischen Bedrohung mit der Rhetorik der Bundeskanzlerin zu Russland in Deutschland.

83 Giegerich/Terhalle 2021, sowie deren Beitrag in diesem Heft.

84 Vgl. Nachtwei 2019, Brockmeier/Rotman 2019.

85 Hierzu vgl. Krause/Mallory 2014; Krause/Jost 2015, Schroeder 2015, Schroeder 2019, Patz 2019, Barbin 2020. 
Jahren aufgebauten russischen Mittelstreckenrüstung steht, die ohne weiteres von konventionell auf nuklear umgestellt werden kann. Vor allem die Bedrohung durch Mittelstreckenwaffen ähnelt in gewisser Weise jener gegen Ende der 70er Jahre, als die Sowjetunion ihre Drohkulisse von Gefechtsfeld- auf Mittelstreckenwaffen erweiterte. Der damalige Bundeskanzler Helmut Schmidt verstand die Zielrichtung dieser Rüstungspolitik sehr richtig und reagierte prompt. Die bisherige deutsche Bundeskanzlerin Angela Merkel schweigt sich aus über die Bedrohungslage.

Dieses Problem ist allerdings nicht auf Deutschland beschränkt. Es scheint, wie der britische Wissenschaftler Hew Strachan feststellte, ein allgemeines Phänomen westlicher Länder zu sein: Westliche Gesellschaften und die Öffentlichkeit würden von ihren Regierungen viel zu wenig über Fragen nationaler Sicherheit informiert und auch von den Medien nur dürftig informiert. Dies trage dazu bei, dass die Resilienz gegenüber Bedrohungen und die Fähigkeit zur Abschreckung unterminiert werde. ${ }^{86}$ Die Abneigung, über militärische Bedrohungen zu sprechen und über Möglichkeiten, diese durch Verteidigungsanstrengungen und Abschreckungspolitik zu neutralisieren, ist nicht neu. Sie war eine Begleiterscheinung der NATO seit ihrer Gründung. Erst in den 60er Jahren nach Verabschiedung des oben erwähnten Harmel-Reports änderte sich die Situation. Das bedeutet, dass eine Politisierung der Verteidigungspolitik notwendig ist. Sie muss sich sowohl mit der Bedrohungsanalyse befassen (warum verfolgt Russland eine derart feindselige und militarisierte Politik gegenüber seinen Nachbarstaaten und warum bedroht es erneut europäische Staaten mit Nuklearwaffen?) als auch nach Antworten suchen - die von Verteidigungsmaßnahmen und Abschreckung bis hin zu positiven Anreizen und Dialogen reichen. Noch liegt Deutschland eine solche Politisierung fern. Man findet sich stattdessen ab mit friedenspolitischen Leerformeln (wie „mit militärischen Mitteln lassen sich keine Probleme lösen“), die in den vergangenen Jahren erkennbar keine Wirkung gezeigt haben.

Des Weiteren besteht ein politisch wichtiger Zusammenhang zwischen der Investitionsbereitschaft in die konventionelle Bündnisverteidigung gegen Russland und möglichen Herausforderungen im nuklearen Bereich, die Deutschlands Politik und auch die der Allianz überfordern würden. Die russische nukleare Escalate-to-DeescalateStrategie (oder Strategie der nuklearen Eskalationsdominanz) wird nur dann eine ernsthafte Gefahr, wenn die Bündnisverteidigung der baltischen Staaten so schwach ist, dass sich Russland ausrechnen kann, mit einem fait

86 Strachan 2020, 72. accompli davonzukommen. Bei ausreichenden Defensivmaßnahmen der Allianz hingegen wird das nicht der Fall sein.

Das heißt: Um eine Krise zu vermeiden, in der die nuklearen Dilemmata wirksam werden können, sollte gerade die Bundesregierung sich an der konventionellen Verteidigung des Bündnisses im Ostseeraum beteiligen. Entsprechende Beschlüsse und Zusagen wurden seit 2014 gemacht und lassen erkennen, dass es die NATO ernst meint mit einer stärkeren Betonung der Verteidigung. ${ }^{87}$ Andererseits ist die bisherige Bundesregierung ihren gegenüber der NATO eingegangenen Verpflichtungen bis heute nicht nachgekommen, weil vor allem ein Koalitionspartner sich ihnen entziehen will und der andere nicht vehement genug auf ihre Einhaltung drängt. ${ }^{88}$ Stattdessen verlangt die Politik gern mehr strategische Autonomie in Europa, Deutschlands Sozialdemokraten fordern gar eine europäische Armee. Aber sobald es in die Details geht, erweist sich, dass eine europäische strategische Autonomie - durch eine europäische Armee oder intensive Kooperation - enorme Investitionen in Verteidigungs- und Offensivfähigkeiten beanspruchen würde, die vermutlich genau jene ablehnen werden, die heute diese Autonomie fordern. ${ }^{89}$

Eine weitere Nagelprobe steht an, wenn die Modernisierung der DCA-Flugzeuge der Bundeswehr beschlossen werden muss. Die derzeit im Rahmen der nuklearen Teilhabe vorgesehenen Tornado-Flugzeuge wären nach Plänen der Bundeswehr demnächst durch eigens nuklear zertifizierte F-18 Flugzeuge zu ersetzen, die es zu beschaffen gilt. Politische Parteien, Medien und Nichtregierungsorganisationen werden mit Nachdruck versuchen, die Entscheidung auszusetzen. Doch von dieser hängt auch ab, ob die NATO eine anfänglich begrenzte regionale militärische Bedrohung noch durch ausreichende Abschreckung entkräften kann. Wer immer die neue Bundesregierung führt, wird eine schwierige Aufgabe zu lösen haben. Man kann nur hoffen, dass diese Person und die sie unterstützenden politischen Kräfte die nötige Einsicht und Courage mitbringen, das zu tun, was im Interesse dieses Landes, seiner Freunde und Verbündeten das Richtige ist.

87 Meyer zum Felde 2018, siehe auch Masuhr 2020.

88 Meyer zum Felde 2020.

89 Vgl. Barrie/Barry/Boyd/Childs/Giegerich 2019, Posen 2020, Barrie/ Barry/Boyd/Childs/Giegerich 2021, Heisbourg 2021, Brooks/Meijer 2021, Posen 2021. 


\section{Literatur}

Adomeit, Hannes (2021): Russland und der Westen - von „strategischer Partnerschaft" zur strategischen Gegnerschaft, Sirius - Zeitschrift für strategische Analysen, 5 (2), 107-124

Arkin, William M./Fieldhouse, Richard W. (1985): Nuclear Battlefield. Global Links in the Arms Races. Cambridge, Mass: Ballinger

Barbin, Jéronimo L.S. (2020): Antworten auf die Asymmetrie. Westliche Militärdoktrinen zur Aufstandsbekämpfung im Vergleich, in: Jochen Maurer/Martin Rink (Hrsg.): Einsatz ohne Kriege. Die Bundeswehr nach 1990 zwischen politischem Auftrag und militärischer Wirklichkeit. Göttingen: Vandenhoeck und Ruprecht, 173-198

Barrie, Douglas/Barry, Ben/Boyd, Henry/Childs, Nick/Giegerich, Bastian (2019): Defending Europe. Scenario-based Capability Requirements for NATO's European Members. London: IISS

Barrie, Douglas/Barry, Ben/Boyd, Henry/Childs, Nick/Giegerich, Bastian (2021): Europe's Defence Requires Offence, Survival, 63 (1), 19-24

Becker, Abraham S. (1982): Der Vorrang militärischer Anstrengungen der Sowjetunion unter den politischökonomischen Bedingungen der 80er Jahre, in: Uwe Nerlich/ Falk Bomsdorf (Hrsg.): Die Einhegung der sowjetischen Macht. Baden-Baden: Nomos Verlag, 39-81

Bergeron, James Henry (2018): Die Dynamik der Abschreckung, Sirius - Zeitschrift für strategische Analysen, 2 (1), 21-31

Bethe, Hans A. (1969). Countermeasures to ABM-Systems, in: Chayes/Wiesner 1969, 130-143

Bluth, Christoph (1995): Britain, Germany, and Western Nuclear Strategy. Oxford und New York: Oxford University Press

Boulégue, Mathieu (2018): Fünf Anmerkungen zu Zapad 2017, Sirius - Zeitschrift für strategische Analysen, 1 (4), 387-388

Bozo, Frederic (1998): Détente versus Alliance: France, the United States and the politics of the Harmel report (1964-1968), Contemporary European History, 7 (3), 343-360

Bracken, Paul (1983): The Command and Control of Nuclear Forces. New Haven und London: Yale University Press

Brauß, Heinrich/Krause, Joachim (2019): Was will Russland mit den vielen Mittelstreckenwaffen?, Sirius - Zeitschrift für strategische Analysen, 3 (2), 154--166

Brennan, Donald G., Hrsg. (1961): Arms Control, Disarmament, and National Security. New York: George Braziller Publ.

Brockmeier, Sarah/Rotmann Philipp (2019): Weit genug gedacht? Drei Jahre PeaceLab im Rückblick, Sirius - Zeitschrift für strategische Analysen, 3 (4), 378-395

Brodie, Bernard (1958): The Anatomy of Deterrence. Santa Monica: Cal.: the RAND Corporation

Brooks, Stephen G./Meijer, Hugo (2021): Europe cannot Defend itself. The Challenge of Pooling Military Power, Survival, 63 (1), 33-40

Bruusgaard, Kristin Ven (2016): Russian Strategic Deterrence, Survival, 58 (4) 7-26

Bull, Hedley, Hrsg. (1961): The Control of the Arms Race. Disarmament and Arms Control in the Missile Age. New York: Praeger

Charles, Daniel (1987): Nuclear Planning in NATO. Pitfalls of First Use. Cambridge, Mass.: Ballinger

Chayes, Abram/Wiesner, Jerome B., Hrsg. (1969): ABM - an Evaluation of the Decision to Deploy an Antiballistic Missile System. New York und London: Harper and Row
Clark, Mason (2020): Russian Hybrid War. Washington, D.C.: Institute for the Study of War (ISW)

Cohen, Raphael S./Radin, Andrew (2019): Russia's Hostile Measures in Europe. Understanding the Threat. Santa Monica, Cal.: The RAND Corporation

Colby, Elbridge (2021): Deutschland am Scheidepunkt - eine aktive Verteidigungs- und Bündnispolitik ist überfällig, Sirius Zeitschrift für strategische Analysen, 5 (3), 227-238

Cordesman, Anthony H. (1982): Deterrence in the 1980s -Part I. American Strategic Forces and Extended Deterrence. London: International Institute for Strategic Studies (Adelphi Paper 175)

Cornides, Wilhelm (1957): Die Weltmächte und Deutschland. Geschichte der jüngsten Vergangenheit 1945-1955. Tübingen/ Stuttgart: Rainer Wunderlich Verlag/J.B. Metzlersche Verlagsbuchhandlung

Daalder, Ivo (1991): The Nature and Practice of Flexible Response. NATO Strategy and Theater Nuclear Forces since 1967. New York: Columbia University Press

De Wijk, Rob (2018): Die Rolle von Abschreckung im neuen strategischen Umfeld Europas, Sirius - Zeitschrift für strategische Analysen, 2 (1), 3-20

DeLauer, Richard D. (1986): Emerging Technologies and their Impact on the Conventional Deterrent, in: Andrew J. Pierre (Hrsg.): The Conventional Defense of Europe. New York: Council on Foreign Relations

Delpech, Thérèse (2012): Nuclear Deterrence in the 21st Century: Lessons from the Cold War for a New Era of Strategic Piracy. Santa Monica, Cal.: The RAND Corporation

Dick, Charles (2019): Russian Ground Force Posture towards the West. London: Royal Institute of International Affairs

Estonian Foreign Intelligence Service (2021): International Security and Estonia - Report on Russia. Tallinn: Estonian Foreign Intelligence Service

Fainberg, Sarah (2017): Russian Spetsnaz, Contractors and Volunteers in the Syrian Conflict. Paris: Institut français des relations internationales

Feld, Bernard T. (1969): The ABM and Arms Control, in: Chayes/ Wiesner 1969, 187-192

Fewtrell, David (1982): The Soviet Economic Crisis. Prospects for the Military and the Consumer. London: International Institute for Strategic Studies (Adelphi Paper 167)

FitzGerald, Mary C. (1987): Marshal Ogarkov and the New Revolution in Soviet Military Affairs. Alexandria, Va.: Center for Naval Analyses

Forss, Stefan/Kiianlinna, Lauri/Inkinen, Pertti/Hult, Heikki (2013): The Development of Russian Military Policy and Finland. Tampere: National Defence University, Department of Strategic and Defence Studies, Research Reports No 49

Fruehling, Stephan/Lasconjarias, Guillaume (2016): NATO, A2/AD and the Kaliningrad Challenge, Survival, 58 (2), 95-116

Fuhrhop, Pia/Kühn, Ulrich/Meier, Oliver (2020): Teilhabe als Chance. Wie Deutschland atomwaffenfrei werden kann und die Sicherheit Europas dabei noch gestärkt wird, Internationale Politik, 75 (4), 62-66

Galeotti, Mark (2016): Heavy Metal Diplomacy. Russia's Political Use of its Military in Europe since 2014. London und Berlin: European Council on Foreign Relations

Galeotti, Mark (2019): Russian Political War. Moving Beyond the Hybrid. Abingdon: Routledge 
Garejew, Machmut A. (1996): Konturen des bewaffneten Kampfes der Zukunft. Ein Ausblick auf das Militärwesen in den nächsten 10 bis 15 Jahren. Baden-Baden: Nomos Verlag

Giegerich, Bastian/Terhalle, Maximilian (2021): Verteidigung ist Pflicht - Deutschlands außenpolitische Kultur muss strategisch werden. Teil 1, Sirius - Zeitschrift für strategische Analysen, 5 (3), 203-226

Giles, Keir (2016): Russia's New Tools for Confronting the West. Continuity and Innovation in Moscow's Exercise of Power. London: Royal Institute of International Affairs

Hackett, James (2021): Die Modernisierung der russischen Streitkräfte, in: Sirius - Zeitschrift für strategische Analysen, 5 (2), 125-139

Haftendorn, Helga (1983): Sicherheit und Entspannung. Zur Außenpolitik der Bundesrepublik Deutschland 1955-1982. Baden-Baden: Nomos Verlag

Haftendorn, Helga (1992): Entstehung und Bedeutung des Harmel-Berichts der NATO von 1967, Vierteljahreshefte für Zeitgeschichte, 40 (2), 169-221

Haftendorn, Helga (1994): Kernwaffen und die Glaubwürdigkeit der Allianz. Die NATO-Krise von 1966/67. Baden-Baden: Nomos

Halverson, Thomas E. (1995): The Last Great Nuclear Debate. NATO and Short Range Nuclear Weapons in the 1980s. Houndmills: Palgrave Macmillan

Hartmann, Rüdiger/Heydrich, Wolfgang/Meyer-Landrut, Nikolaus (1994): Der Vertrag über konventionelle Streitkräfte in Europa. Baden-Baden: Nomos Verlag

Heisbourg, François (2021): Europe can Afford the Cost of Autonomy, Survival, 63 (1), 25-31

Heuser, Beatrice (1997): NATO, Britain, France and the FRG. Nuclear Strategies and Forces for Europe, 1949-2000. Houndmills: Palgrave Macmillan

Heuser, Beatrice (2006): Alliance of Democracies and Nuclear Deterrence, in: Mastny/Holtsmark/Wenger 2006, 193-216

Heymont, Irving (1966): The NATO bilateral nuclear forces, Orbis, 9 (4), 1025-1041

Hinz, Stefan C. P. (2019): Der INF-Vertrag - eine (fast) postmortale Betrachtung, Sirius - Zeitschrift für strategische Analysen, 3 (2), 167-172

Hoppe, Christoph (1993): Zwischen Teilhabe und Mitsprache. Die Nuklearfrage in der Allianzpolitik Deutschlands. Baden-Baden: Nomos Verlag

Johnson, Dave (2019): General Gerasomov über die Entwicklungslinien der russischen Militärstrategie - eine Analyse, Sirius - Zeitschrift für strategische Analysen, 3 (3), 255-261

Kamp, Karl Heinz (2019): Das strategische Langfristproblem der NATO, Sirius - Zeitschrift für strategische Analysen, 3 (2), 129-135

Kamp, Karl-Heinz (2007): Die NATO und die nukleare Abschreckung, in: Henning Riecke (Hrsg.): Die Transformation der NATO. Baden-Baden: Nomos Verlag, 93-106

Kamp, Karl-Heinz (2018): Die neue Nuklearausrichtung der NATO, Sirius - Zeitschrift für strategische Analysen, 1 (4), 358-365

Kanet, Roger, Hrsg. (2017): The Russian Challenge to the European Security System. Basingstoke: Palgrave MacMillan

Karber, Phillip A. (1982): Die konventionellen Kräfteverhältnisse in Europa 1965-1980, in: Uwe Nerlich und Falk Bomsdorf (Hrsg.): Sowjetische Macht und westliche Verhandlungspolitik im Wandel militärischer Kräfteverhältnisse. Baden-Baden 1982, 49-133
Kaufmann, William W. (1964): The McNamara Strategy. New York und London: Harper \& Row

Kelleher, Catherine M. (1975): Germany and the Politics of Nuclear Weapons. New York: Columbia University

Kelleher, Catherine M. (1988): NATO Nuclear Operations, Survival, 30 (1), 59-78

Kissinger, Henry A. (1957): Nuclear Weapons and Foreign Policy. New York: Harper (Council on Foreign Relations)

Kissinger, Henry A. (1965): Was wird aus der westlichen Allianz? Wien/Düsseldorf: Econ Verlag

Klain, Robert M./Lundqvist, Stefan/Sumangil, Ed/Pettersson, Ulrica (2019): Baltics Left to Bang. The Role of NATO with Partners in Denial-Based Deterrence. Stockholm: Strategic Forum

Kramer, Franklin/Binnendijk, Hans (2018): Meeting the Russian Conventional Challenge. Effective Deterrence by Prompt Reinforcement. Washington, D.C.: Atlantic Council

Krause, Joachim (1988): Prospects for Conventional Arms Control in Europe. New York: Institute for East-West Security Studies

Krause, Joachim (2015): Threat Scenarios, Risk Assessments, and the Future of Nuclear Deterrence, in von Hlatky/Wenger 2015, 19-39

Krause, Joachim (2021a): Die transatlantischen Beziehungen nach den US-Wahlen vom November 2020. Perspektiven der Wiederannäherung, Zeitschrift für Parlamentsfragen, 52 (2), 338-357

Krause, Joachim (2021b): Deutsche Rüstungskontrollpolitik im strategischen Niemandsland, Sirius - Zeitschrift für strategische Analysen, 5 (2), 157-164

Krause, Joachim/Jost, Jannis (2015): Stabilisierungseinsätze zwischen außenpolitischem Risiko und strategischer Notwendigkeit, in: Schroeder/Hansen 2015, 45-63

Krause, Joachim/Magiera-Krause, Christiane, Hrsg. (1992): Dokumentation zur Abrüstung und Rüstungskontrolle. Band 24 -1987/1988. St. Augustin: Ziegler

Krause, Joachim/Magiera-Krause, Christiane, Hrsg. (1997): Dokumentation zur Abrüstung und Sicherheit. Band 25 1989-1992. Sankt Augustin: Akademia Verlag

Krause, Joachim/Mallory, Charles King, IV, Hrsg. (2014): Afghanistan, Pakistan and Strategic Change. Adjusting Western regional policy. London und New York: Routledge

Kristensen, Hans M. (2015): Nuclear Weapons in NATO's Deterrence Posture. Status Quo or Change?, in: von Hlatky/Wenger 2015, 135-151

Kristensen, Hans M. (2018): Russland modernisiert Kernwaffendepot im Bezirk Kaliningrad, Sirius - Zeitschrift für strategische Analysen, 2 (4), 398-400

Kristensen, Hans M./Korda, Matt (2021). United States Nuclear Weapons, 2021. Bulletin of the Atomic Scientists, 77 (1), 43-63

Kroenig, Matthew (2018): Russlands Nuklearstrategie gegenüber Europa - wie organisiert man Abschreckung gegen Deeskalation mit nuklearen Schlägen?, Sirius - Zeitschrift für strategische Analysen, 2 (4), 323-338

Larsen, Jeffrey A. (2015): US Extended Deterrence and Europe. Time to Consider Alternative Structures?, in: von Hlatky/Wenger 2015, 41-69

Lautsch, Siegfried (2013): Kriegsschauplatz Deutschland. Erfahrungen und Erkenntnisse eines NVA-Offiziers. Potsdam Zentrum für Militärgeschichte und Sozialwissenschaften der Bundeswehr 
Lavrov, Anton (2018): Russian Military Reforms from Georgia to Syria. Washington, D.C.: Center for Strategic and International Studies Lunák, Petr (2001): Planning for Nuclear War - The Czechoslovak War Plan of 1964, Cold War International History Project Bulletin, 12-13, 289-298

Lunák, Petr (2006): War Plans from Stalin to Brezhnev. The Czechoslovak pivot, in: Mastny/Holtsmark/Wenger 2006, 72-93

Lutsch, Andres (2020): Westbindung oder Gleichgewicht? Die nukleare Sicherheitspolitik der Bundesrepublik Deutschland zwischen Atomwaffensperrvertrag und NATO-Doppelbeschluss. Berlin: DeGruyter Oldenbourg

Mahncke, Dieter (1972): Nukleare Mitwirkung. Die Bundesrepublik Deutschland in der Atlantischen Allianz 1949-1970. Berlin: DeGruyter

Major, Claudia (2019): Die Rolle der NATO für Europas Verteidigung. Stand und Optionen zur Weiterentwicklung aus deutscher Perspektive. Berlin: Stiftung Wissenschaft und Politik

Mallory, Charles King, IV (2019): Domänenübergreifende Abschreckung. Eine neue Herausforderung westlicher Sicherheitspolitik, Sirius - Zeitschrift für strategische Analysen, 3 (1), 45-64

Mandelbaum, Michael (1984): The Anti-Nuclear Weapons Movements, PS: Political Science \& Politics, 17 (1), 24-32

Martin, James J. (1982): Die nuklearen Kräfteverhältnisse in Europa 1970-1980, in: Uwe Nerlich und Falk Bomsdorf (Hrsg.): Sowjetische Macht und westliche Verhandlungspolitik im Wandel militärischer Kräfteverhältnisse. Baden-Baden 1982, 135-183

Masala, Carlo (2016): Weltunordnung. Die globalen Krisen und das Versagen des Westens. München: C H Beck

Mastny, Vojtech (2006): Imagining War in Europe. Soviet Strategic Planning, in: Mastny/Holtsmark/Wenger 2006, 15-45

Mastny, Vojtech/Holtsmark, Sven G./Wenger, Andreas, Hrsg. (2006): War Plans and Alliances in the Cold War. Threat Perceptions in the East and West. Abingdon und New York: Routledge

Masuhr, Niklas (2020): Operative Anpassungen von NATOStreitkräften seit der Krim - Muster und Divergenzen, Sirius - Zeitschrift für strategische Analysen, 4 (2), 170-183

McNamara, Robert S. (1970): Die Verteidigung des Westens. Bedrohung und Abwehr. München: Deutscher Taschenbuch Verlag

Meyer zum Felde, Rainer (2018): Abschreckung und Dialogbereitschaft. Der Paradigmenwechsel der NATO seit 2014, Sirius - Zeitschrift für strategische Analysen, 2 (2), 101-117

Meyer zum Felde, Rainer (2020): Deutsche Verteidigungspolitik - Versäumnisse und nicht eingehaltene Versprechen, Sirius - Zeitschrift für strategische Analysen, 4 (3), 315-332

Meyer, Stephen M. (1984a): Soviet Theatre Nuclear Forces. Part I: Development of Doctrine and Objectives. London: International Institute for Strategic Studies (Adelphi Paper 187)

Meyer, Stephen M. (1984b): Soviet Theatre Nuclear Forces. Part II: Capabilities and Implications. London: International Institute for Strategic Studies (Adelphi Paper 188)

Mützenich, Ralf (2020): Nukleare Teilhabe - ein überholtes Konzept, WeltTrends, 167, 68-70

Nachtwei, Winfried (2019): Lehren aus deutschen Krisenengagements gibt es reichlich - aber auch Lernfortschritte?, Sirius - Zeitschrift für strategische Analysen, 3 (4), 362-377
Nerlich, Uwe (2020): Auf der Suche nach politischer Rationalität nuklearer Abschreckung, Sirius - Zeitschrift für strategische Analysen, 4 (4), 399-409

Nielsen, Harald (1998): Die DDR und die Kernwaffen. Die nukleare Rolle der Nationalen Volksarmee im Warschauer Pakt. Baden-Baden: Nomos Verlag

Nilsen, Thomas (2018): Russland erweitert im großen Umfang seine Kernwaffenlager auf der Kola Halbinsel, Sirius - Zeitschrift für strategische Analysen, 2 (4), 401-405

Palmer, Diego Ruiz (2018): Theatre Operations, High Commands and Large Scale Exercises in Soviet and Russian Military Practice. Rom: NATO Defence College

Patz, Christian (2019): Peacekeeping Labor Mali. Deutschland und der MINUSMA Einsatz, Sirius - Zeitschrift für strategische Analysen, 3 (4), 339-361

Petersen, Philip A./Hines, John (1983): The Conventional Offensive in Soviet Theatre Strategy, Orbis, 27 (3), 695-739

Posen, Barry R. (2020): Europe can Defend itself, Survival, 62 (6), 7-34

Posen, Barry R. (2021): In Reply. To Repeat, Europe can Defend itself, Survival, 63 (1), 41-49

Richardson, James L. (1967): Deutschland und die NATO. Köln und Opladen: Westdeutscher Verlag

Roberts, Brad (2016): The Case for U.S. Nuclear Weapons in the 21st Century. Stanford, Cal.: Stanford University Press

Roberts, Brad (2020): Neue Herausforderungen erfordern neue Ideen: Elemente einer Theorie des Sieges in modernen strategischen Konflikten, Sirius - Zeitschrift für strategische Analysen, 4 (4), 410-434

Roberts, Brad (2021a): Deutschland und die Nukleare Teilhabe der NATO. Berlin: Bundesakademie für Sicherheitspolitik (Arbeitspapier)

Roberts, Brad (2021b): Orienting the 2021 Nuclear Posture Review, The Washington Quarterly, 44 (2), 123-142

Ruehle, Michael (2020): Die (unvollkommene) Rückkehr der Abschreckung, Sirius - Zeitschrift für strategische Analysen, 4 (4), 387-398

Rühl, Lothar (1987): Mittelstreckenwaffen in Europa. Ihre Bedeutung in Strategie, Rüstungskontrolle und Bündnispolitik. Baden-Baden: Nomos

Rynning, Sten (2017): The Divide: France, Germany and political NATO, International Affairs, 93 (2), 267-289

Schelling, Thomas (1966): Arms and Influence. New Haven, Conn.: Yale University Press

Schroeder, Robin (2015): Über das Zusammenwirken von Sicherheit, Wiederaufbau und Entwicklung im Kontext von Stabilisierungseinsätzen, in: Schroeder/Hansen 2015, 65-90

Schroeder, Robin (2019): Zivil-militärische Stabilisierung als Handlungsfeld im internationalen Krisenmanagement, Sirius Zeitschrift für strategische Analysen, 3 (4), 329-338

Schroeder, Robin/Hansen, Stefan, Hrsg. (2015): Stabilisierungseinsätze als gesamtstaatliche Aufgabe. Erfahrungen und Lehren aus dem deutschen Afghanistaneinsatz zwischen Staatsaufbau und Aufstandsbewältigung (COIN). Baden-Baden: Nomos Verlag

Schulte, Paul (2015): NATO's Protracted Debate over Nuclear Weapons, in: von Hlatky/Wenger 2015, 107-133

Shlapak, David A./Johnson, Michael W. (2016): Reinforcing Deterrence on NATO's Eastern Flank: Wargaming the Defense of the Baltics. Santa Monica, Cal.: The RAND Corporation 
Shulman, Marshall D. (1969): The Effect of ABM on U.S.-Soviet Relations, in: Chayes/Wiesner 1969, 153-159

Sokolsky, Richard (2017): The New NATO-Russia Military Balance. Implications for European Security. Washington, D.C.: Carnegie Endowment

Spohr, Kristina (2016): Helmut Schmidt. Der Weltkanzler. Darmstadt: Theiss-WBG

Strachan, Hew (2020): Strategy and Democracy, Survival, 62 (2), 51-82

Stratmann, K.-Peter (1981): NATO-Strategie in der Krise? Militärische Optionen von NATO und Warschauer Pakt in Mitteleuropa. Baden-Baden: Nomos Verlag

Stromseth, Jane E. (1988): The Origins of Flexible Response. NATO's Debate over Strategy in the 1960s. New York: St. Martin's Press

Thomson, James A. (2018): Abschreckung einst und heute, Sirius Zeitschrift für strategische Analysen, 2 (1), 32-41

Thränert, Oliver (2018): Präsident Trumps Nuclear Posture Review, Sirius - Zeitschrift für strategische Analysen, 2 (2), 158-161

Thränert, Oliver/Kartchner, Kerry M. (2015): From Offense to Defense? Extended Deterrence and Missile Defense, in: von Hlatky/Wenger 2015, 155-176
Vershbov, Alexander R./Breedlove, Philip M. (2019): Permanent Deterrence. Enhancements to the U.S. Military Presence in North Central Europe. Washington, D.C.: The Atlantic Council

Vigor, Peter (1983): Soviet Blitzkrieg Theory. New York: St. Martin's Press

Von Hlatky, Stéfanie/Wenger, Andreas, Hrsg. (2015): The Future of Extended Deterrence. The United States, NATO and Beyond. Washington, D.C.: The Georgetown University Press

Wilk, Andrzej (2021): By armour and by fear: the Russian army on the western flank. Warschau: Centre for Eastern Studies (OSW)

Windsor, Philip (1971): Germany and the Management of Détente. London: Chatto \& Windus

Wohlstetter, Albert (1959): The Delicate Balance of Terror, Foreign Affairs, 37 (2), 212-234

Woolf, Amy F. (2018): U.S. Strategic Nuclear Forces: Background, Developments, and Issues. Washington, D.C.: Congressional Research Service

Woolf, Amy F. (2021): Non-Strategic Nuclear Weapons. Washington, D.C.: Congressional Research Service

Zdanavičius, Liudas/Czekaj, Matthew, Hrsg. (2015): Russia's Zapad 2013 Military Exercise. Lessons for Baltic Regional Security. Washington, D.C. und Riga: Jamestown Foundation/National Defence Academy of Latvia. 